\title{
On the Variable-order Fractional Laplacian Equation with Variable Growth on $\mathbb{R}^{N}$
}

\author{
Nguyen Van Thin
}

\begin{abstract}
The aim of this paper is to study the existence of solutions to the variableorder fractional Laplacian as follows:

$$
(-\Delta)^{s(\cdot)} u+V(x) u=\lambda f(x, u) \quad \text { in } \mathbb{R}^{N},
$$

where $\lambda>0$ is a parameter, $N \geq 1,(-\Delta)^{s(\cdot)}$ is the variable-order fractional Laplacian operator with $s(\cdot): \mathbb{R}^{N} \times \mathbb{R}^{N} \rightarrow(0,1)$ is continuous function with $N>2 s^{+} \geq 2 s(x, y)$ for all $(x, y) \in \mathbb{R}^{N} \times \mathbb{R}^{N}$, and $f$ has variable growth and $V$ satisfies some suitable assumptions. Using Mountain Pass Theorem, Fountain Theorem and Genus theory, we obtain the existence of weak solutions to above problem.
\end{abstract}

\section{Introduction and main results}

The aim of this paper is to study the existence of solutions to the variable-order fractional Laplace as follows:

$$
(-\Delta)^{s(\cdot)} u+V(x) u=\lambda f(x, u) \text { in } \mathbb{R}^{N},
$$

where $\lambda>0$ is a parameter, $N \geq 1,(-\Delta)^{s(\cdot)}$ is the variable-order fractional Laplacian operator with $s(\cdot): \mathbb{R}^{N} \times \mathbb{R}^{N} \rightarrow(0,1)$ is a continuous functions and symmetric with $N>2 s^{+} \geq 2 s(x, y)$ for all $(x, y) \in \mathbb{R}^{N} \times \mathbb{R}^{N}$, and $f$ has variable growth and $V: \mathbb{R}^{N} \rightarrow$ $(0,+\infty)$ is a continuous function satisfying some suitable assumptions. The variable-order fractional Laplace $(-\Delta)^{s(\cdot)}$ is defined as follows: for each $x \in \mathbb{R}^{N}$,

$$
(-\Delta)^{s(\cdot)} \varphi(x)=2 \mathrm{P} . \mathrm{V} \cdot \int_{\mathbb{R}^{N}} \frac{\varphi(x)-\varphi(y)}{|x-y|^{N+2 s(x, y)}} d y=2 \lim _{\varepsilon \rightarrow 0} \int_{\mathbb{R}^{N} \backslash B_{\varepsilon}(x)} \frac{\varphi(x)-\varphi(y)}{|x-y|^{N+2 s(x, y)}} d y
$$

along any $\varphi \in C_{0}^{\infty}\left(\mathbb{R}^{N}\right)$, where $\mathrm{P}$. V. denotes by the Cauchy principle value and $B_{\varepsilon}(x)=$ $\left\{y \in \mathbb{R}^{N}:|y-x|<\varepsilon\right\}$. When $x=0$, we write $B_{\varepsilon}$ instead of $B_{\varepsilon}(0)$. If $s(\cdot) \equiv$ constant, then $(-\Delta)^{s(\cdot)}$ reduced to the usual fractional Laplace. This operator is introduced by the works in $3,4,11,23$, where the fractional Sobolev space with variable exponent is defined

Received July 9, 2020; Accepted June 21, 2021.

Communicated by François Hamel.

2020 Mathematics Subject Classification. 35A15, 35J60, 35R11, 35S15.

Key words and phrases. integrodifferential operators, variable-order fractional Laplacian, mountain pass theorem, fountain theorem, genus theory. 
and some properties are proved. In [11], the authors established the compact embedding in fractional Sobolev space $W^{s, q(\cdot), p(\cdot)}(\Omega)$ and using it to study the weak solution of a boundary problem. The density properties of $W^{s, q(\cdot), p(\cdot)}(\Omega)$ is studied in [4] with some suitable assumptions of function $p$. These type of nonlocal operators arises in a quite natural way in many different applications such as optimization, finance, phase transitions, stratified materials, anomalous diffusion, crystal dislocation, soft thin films, semipermeable membranes, flame propagation, conversion laws, ultra-relativistic of quantum mechanics, quasi-geotrophic flows, multiple scattering, minimal surface, materials science and water waves. We refer the readers to $14,17,18$ for more motivation and details application of nonlocal problem. For topics about $p(x)$-Laplace equation, we refer the readers to [1,9, 13 and references therein for more details. In [2,6], the authors study the nonlocal eigenvalue problem for fractional $p(\cdot)$-Laplacian by using variational techniques. The fractional order derivatives has long history that prompted L'Hospital in 1695 to ask Leibniz. Since that time, there are many kinds of definitions for fractional order derivatives are defined by Riemann-Liouville, Caputo, Grünwald-Letnikov, etc. In [16], Lorenzo-Hartley studied the nonlinear diffusion processes with fractional variable order derivatives. In particular, some diffusion processes reacting to temperature changes may be better described by using variable order derivatives in a nonlocal integro-differential operator. Leopold in 15 considered multifractional pseudodifferential models in the representation of heterogeneous local behaviors and studied the solutions to such models in fractional Besov spaces of variable order in $\mathbb{R}^{N}$. Kikuchi and Negoro in $[12$ obtained the conditions that general pseudodifferential operators on fractional Sobolev spaces of variable order in $\mathbb{R}^{N}$ form a Feller semigroup which has a transition density. Especially, Ruiz-Medina et al. in 20 studied some Gaussian processes defined by elliptic pseudodifferential equations, in which the covariance function of these random processes defines the inner product of a fractional Sobolev space of variable order.

For $g: \mathbb{R}^{N} \rightarrow \mathbb{R}$, we denote $g^{-}=\inf _{x \in \mathbb{R}^{N}} g(x), g^{+}=\sup _{x \in \mathbb{R}^{N}} g(x)$. We define

$$
C_{+}\left(\mathbb{R}^{N}\right)=\left\{g \in C\left(\mathbb{R}^{N}\right): 1<\inf _{x \in \mathbb{R}^{N}} g(x) \leq \sup _{x \in \mathbb{R}^{N}} g(x)<+\infty\right\} .
$$

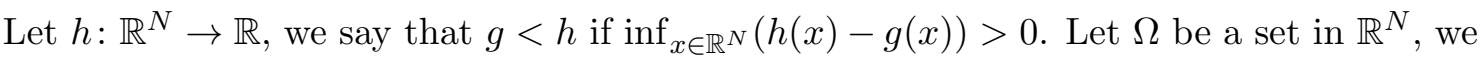
denote meas $(\Omega)$ by the Lebesgue measure of the set $\Omega$.

Inspired by the above works, we assume that $s, V$ satisfy the following conditions:

$\left(\mathrm{S}_{1}\right) 0<s^{-} \leq s^{+}<1$

$\left(\mathrm{S}_{2}\right) s$ is a symmetric function, that is, $s(x, y)=s(y, x)$ for all $(x, y) \in \mathbb{R}^{N} \times \mathbb{R}^{N}$;

$\left(\mathrm{V}_{1}\right) V: \mathbb{R}^{N} \rightarrow \mathbb{R}$ is a continuous function such that $V^{-}>0 ;$ 
$\left(\mathrm{V}_{2}\right) \operatorname{meas}\left(x \in \mathbb{R}^{N}: V(x)<c\right)<+\infty$ for any $c>0$;

$\left(\mathrm{V}_{3}\right)$ There exists $h>0$ such that $\lim _{|y| \rightarrow+\infty} \operatorname{meas}\left(x \in B_{h}(y): V(x)<c\right)=0$ for any $c>0$.

Now, we give some assumptions on the continuous function $f: \mathbb{R}^{N} \times \mathbb{R} \rightarrow \mathbb{R}$ as follows:

$\left(\mathrm{f}_{1}\right)$ There exists $a>0$ such that $|f(x, t)| \leq a\left(1+|t|^{q(x)-1}\right)$ for all $(x, t) \in \mathbb{R}^{N}$, where $q \in C_{+}\left(\mathbb{R}^{N}\right)$ is a uniformly continuous function such that $q(x)>2$ for all $x \in \mathbb{R}^{N}$ and $\inf _{x \in \mathbb{R}^{N}}\left(2_{s}^{*}(x)-q(x)\right)>0$, where $2_{s}^{*}(x)=\frac{2 N}{N-2 s(x, x)}$.

$\left(\mathrm{f}_{2}\right) f(x, t)=o(t)$ as $t \rightarrow 0$ uniformly for all $x \in \mathbb{R}^{N}$.

$\left(\mathrm{f}_{1}^{\prime}\right)$ There exist measure functions $\gamma_{1}, \ldots, \gamma_{m}, h_{0}, h_{1}, \ldots, h_{m}$ satisfying

$$
1<\gamma_{1}<\gamma_{2}<\cdots<\gamma_{m}<2
$$

where $\left(2 / \gamma_{i}\right)^{+} \geq\left(2 / \gamma_{i}\right)^{-}>1$ and $h_{0}(x) \in L^{1}\left(\mathbb{R}^{N}\right), h_{i}(x) \in L^{2 /\left(2-\gamma_{i}(x)\right)}\left(\mathbb{R}^{N}\right), i=$ $1, \ldots, m$ such that

$$
|f(x, t)| \leq\left|h_{0}(x)\right|+\sum_{i=1}^{m}\left|h_{i}(x)\right||t|^{\gamma_{i}(x)-1}
$$

for all $(x, t) \in \mathbb{R}^{N} \times \mathbb{R}$.

(f) There exist measure functions $\gamma_{1}, \ldots, \gamma_{m}, h_{0}, h_{1}, \ldots, h_{m}$ satisfying

$$
1<\gamma_{1}<\gamma_{2}<\cdots<\gamma_{m}<2<q<2_{s}^{*}(x)=2 N /(N-2 s(x, x)),
$$

where $q \in C_{+}\left(\mathbb{R}^{N}\right)$ is a uniformly continuous function such that

$$
\inf _{x \in \mathbb{R}^{N}}\left(2_{s}^{*}(x)-q(x)\right)>0, \quad\left(q / \gamma_{i}\right)^{+} \geq\left(q / \gamma_{i}\right)^{-}>1,
$$

$h_{0}(x) \in L^{q(x) /(q(x)-1)}\left(\mathbb{R}^{N}\right), h_{i}(x) \in L^{\frac{q(x)}{q(x)-\gamma_{i}(x)}}\left(\mathbb{R}^{N}\right), i=1, \ldots, m$ and the constants $h_{m+1}>0, g_{m+1}>0$ such that

$$
|f(x, t)| \leq\left|h_{0}(x)\right|+\sum_{i=1}^{m}\left|h_{i}(x)\right||t|^{\gamma_{i}(x)-1}+g_{m+1}|t|+h_{m+1}|t|^{q(x)-1}
$$

for all $(x, t) \in \mathbb{R}^{N} \times \mathbb{R}$.

Remark 1.1. We see that the continuous function $f$ satisfies the conditions $\left(\mathrm{f}_{1}\right)$ and $\left(\mathrm{f}_{2}\right)$, then for some $\varepsilon>0$, there exists $C_{\varepsilon}>0$ such that

$$
|f(x, t)| \leq \varepsilon|t|+C_{\varepsilon}|t|^{q(x)-1}
$$

for all $(x, t) \in \mathbb{R}^{N} \times \mathbb{R}$. This means that $f$ verifies the condition $(\mathfrak{f})$. Furthermore, if $f$ satisfies the condition $\left(\mathrm{f}_{1}^{\prime}\right)$, then $f$ also verifies the condition $(\mathfrak{f})$. 
$\left(\mathrm{f}_{3}\right)$ There exists $\mu>2$ such that $\inf _{x \in \mathbb{R}^{N}}\left(2_{s}^{*}(x)-\mu\right)>0$ and $r \geq 0$, we have $t f(x, t)-$ $\mu F(x, t) \geq-\rho|t|^{2}-\varphi_{*}(x)$ for all $x \in \mathbb{R}^{N}$ and $|t| \geq r$, where $F(x, t)=\int_{0}^{t} f(x, \tau) d \tau$, $\varphi_{*} \geq 0$ on $\mathbb{R}^{N}$ and $\varphi_{*} \in L^{1}\left(\mathbb{R}^{N}\right)$.

$\left(\mathrm{f}_{4}\right)$ There exists $C_{\mu}>0$ such that $F(x, t) \geq C_{\mu}|t|^{\mu}-P(|t|)$ for all $(x, t) \in \mathbb{R}^{N} \times \mathbb{R}$, where $P$ is a function on $\mathbb{R}$ such that $P(t)=a_{n}|t|^{\alpha_{n}(x)}+\cdots+a_{1}|t|^{\alpha_{1}(x)}+a_{0}$ for all $t \in \mathbb{R}$ and $n \in \mathbb{N}, a_{i} \in \mathbb{R}, i=0, \ldots, n, a_{n} \neq 0$ and $+\infty>\alpha_{n}(x)>\cdots>\alpha_{1}(x)>0$, $\alpha_{n}^{+} \geq \alpha_{n}(x) \geq 2$ for all $x \in \mathbb{R}^{N}$ and $a_{0} \leq 0, a_{i}<0$ if $\alpha_{i} \in(0,2)$ for some $i \in\{1, \ldots, n-1\}$ if $n \geq 2$. Here $\alpha \in(a, b)$ means $a<\alpha<b$. Furthermore, for all $\alpha \in\left\{\alpha_{1}, \ldots, \alpha_{n}\right\}$ such that $2 \leq \alpha(x) \leq \alpha_{n}(x)<\mu$ for all $x \in \mathbb{R}^{N}$, we have $\inf _{x \in \mathbb{R}^{N}}\left(2_{s}^{*}(x)-\alpha(x)\right)>0$ and $\alpha \in C_{+}\left(\mathbb{R}^{N}\right)$ is a uniformly continuous function.

$\left(\mathrm{f}_{5}\right) f(x,-t)=-f(x, t)$ for all $(x, t) \in \mathbb{R}^{N} \times \mathbb{R}$.

$\left(\mathrm{f}_{6}\right)$ There exists $0<\eta<1$ such that $F(x, t) \geq \delta|t|^{2}$ for all $(x, t) \in \mathbb{R}^{N} \times[-\eta, \eta]$, where $\delta>0$.

Remark 1.2. Setting $a>0$, we consider function

$$
F(x, t)=a \int_{0}^{|t|} \tau \sin \tau d \tau+\frac{|t|^{\mu}}{\mu}=a(\sin |t|-|t| \cos |t|)+\frac{|t|^{\mu}}{\mu},
$$

where $\mu>2$ and $\inf _{x \in \mathbb{R}^{N}}\left(2_{s}^{*}(x)-\mu\right)>0$. We have

$$
f(x, t)=F_{t}^{\prime}(x, t)=a \operatorname{sign} t \cdot|t| \sin |t|+|t|^{\mu-2} t .
$$

Then

$$
f(x, t) t-\mu F(x, t)=a|t|^{2} \sin |t|-a \mu(\sin |t|-|t| \cos |t|) .
$$

If we choose $\left|t_{k}\right|=-\frac{\pi}{2}+2 k \pi, k \in \mathbb{Z}^{+}$, then $\left|t_{k}\right| \rightarrow+\infty$ as $k \rightarrow+\infty$ and

$$
f\left(x, t_{k}\right) t_{k}-\mu F\left(x, t_{k}\right) \rightarrow-\infty
$$

as $k \rightarrow \infty$ since $2<\mu<\frac{2 N}{N-2 s^{-}}<+\infty$. Therefore, $f$ does not satisfy AmbrosettiRabinowitz condition. Clearly, $f$ satisfy the conditions $\left(f_{1}\right),\left(f_{2}\right),\left(f_{3}\right)$ and $\left(f_{4}\right)$. Since $|\sin t| \leq 1$ for all $t \in \mathbb{R}$, then

$$
f(x, t) t-\mu F(x, t)=a|t|^{2} \sin |t|-\mu a \int_{0}^{|t|} \tau \sin \tau d \tau \geq-\rho|t|^{2}
$$

for all $(x, t) \in \mathbb{R}^{N} \times R$, where $\rho=a\left(1+\frac{N}{N-2 s^{-}}\right)>0$. Furthermore, we have

$$
F(x, t) \geq \frac{|t|^{\mu}}{\mu}-\frac{a|t|^{2}}{2} \geq \frac{N-2 s^{-}}{2 N}|t|^{\mu}-\frac{a|t|^{2}}{2}
$$

for all $(x, t) \in \mathbb{R}^{N} \times \mathbb{R}$. 
Before starting our results, we recall some useful notations. The fractional Sobolev space $H^{s(\cdot)}\left(\mathbb{R}^{N}\right)$ is defined by the linear space of Lebesgue function $u \in L^{2}\left(\mathbb{R}^{N}\right)$ such that

$$
[u]_{s(\cdot)}=\left(\iint_{\mathbb{R}^{2 N}} \frac{|u(x)-u(y)|^{2}}{|x-y|^{N+2 s(x, y)}} d x d y\right)^{1 / 2}<+\infty .
$$

Then $H^{s(\cdot)}\left(\mathbb{R}^{N}\right)$ is a norm space with

$$
\|u\|_{H^{s(\cdot)\left(\mathbb{R}^{N}\right)}}=\left(\|u\|_{L^{2}\left(\mathbb{R}^{N}\right)}^{2}+[u]_{s(\cdot)}^{2}\right)^{1 / 2}
$$

for all $u \in H^{s(\cdot)}\left(\mathbb{R}^{N}\right)$, where $[u]_{s(\cdot)}$ denotes by the seminorm Gagliardo, that is,

$$
[u]_{s(\cdot)}=\left(\iint_{\mathbb{R}^{2 N}} \frac{|u(x)-u(y)|^{2}}{|x-y|^{N+2 s(x, y)}} d x d y\right)^{1 / 2} .
$$

Therefore $H^{s(\cdot)}\left(\mathbb{R}^{N}\right)$ is a uniformly convex Banach space and $H^{s(\cdot)}\left(\mathbb{R}^{N}\right)$ is a Hilbert space with inner product

$$
\langle u, v\rangle=\int_{\mathbb{R}^{2 N}} \frac{(u(x)-u(y))(v(x)-v(y))}{|x-y|^{N+2 s(x, y)}} d x d y+\int_{\mathbb{R}^{2 N}} u v d x
$$

for all $u, v \in H^{s(\cdot)}\left(\mathbb{R}^{N}\right)$. Hence, it is a reflexive space. Let $W$ be the completion of $C_{0}^{\infty}\left(\mathbb{R}^{N}\right)$ with norm

$$
\|u\|_{W}=\left([u]_{s(\cdot)}^{2}+\|u\|_{2, V}^{2}\right)^{1 / 2}, \quad\|u\|_{2, V}^{2}=\int_{\mathbb{R}^{N}} V(x)|u(x)|^{2} d x
$$

for all $u \in W$. Then $W$ is a Hilbert space, thus it is a uniformly convex Banach space and $W$ is a reflexive space. Note that from $V^{-}>0$, the space $W$ is embedded continuously into $H^{s(\cdot)}\left(\mathbb{R}^{N}\right)$.

Definition 1.3. We say that $u \in W$ is a weak solution of problem (1.1) if

$\iint_{\mathbb{R}^{2 N}} \frac{(u(x)-u(y))(\varphi(x)-\varphi(y))}{|x-y|^{N+2 s(x, y)}} d x d y+\int_{\mathbb{R}^{N}} V(x) u(x) \varphi(x) d x=\lambda \int_{\mathbb{R}^{N}} f(x, u(x)) \varphi(x) d x$ for any $\varphi \in W$.

We assume that the number $\rho$ in the condition $\left(\mathrm{f}_{3}\right)$ satisfy the following condition

$$
\frac{\lambda \rho}{\mu V^{-}}<\frac{1}{2}-\frac{1}{\mu}
$$

Our first result is given as follows:

Theorem 1.4. Assume that the conditions $\left(\mathrm{S}_{1}\right),\left(\mathrm{S}_{2}\right),\left(\mathrm{V}_{1}\right)$ and $\left(\mathrm{V}_{2}\right)$ or $\left(\mathrm{V}_{1}\right)$ and $\left(\mathrm{V}_{3}\right)$, $\left(\mathrm{f}_{1}\right)-\left(\mathrm{f}_{4}\right)$ hold. Then the problem (1.1) admits one nontrivial solution in $W$ for all $\lambda$ satisfying 1.2 . 
Next, we prove the existence of many solutions of problem 1.1 .

Theorem 1.5. Assume that the conditions $\left(\mathrm{S}_{1}\right),\left(\mathrm{S}_{2}\right),\left(\mathrm{V}_{1}\right)$ and $\left(\mathrm{V}_{2}\right)$ or $\left(\mathrm{V}_{1}\right)$ and $\left(\mathrm{V}_{3}\right)$, $(\mathfrak{f}),\left(\mathrm{f}_{3}\right)-\left(\mathrm{f}_{5}\right)$ hold. Then problem (1.1) has infinitely many solutions $\left\{u_{j}\right\} \in W$ whose energy $I\left(u_{j}\right) \rightarrow+\infty$ as $j \rightarrow+\infty$ for all $\lambda$ satisfying 1.2 .

Using Genus theory, we obtain the following result.

Theorem 1.6. Assume that the conditions $\left(\mathrm{S}_{1}\right),\left(\mathrm{S}_{2}\right),\left(\mathrm{V}_{1}\right)$ and $\left(\mathrm{V}_{2}\right)$ or $\left(\mathrm{V}_{1}\right)$ and $\left(\mathrm{V}_{3}\right)$, $\left(\mathrm{f}_{1}^{\prime}\right),\left(\mathrm{f}_{5}\right),\left(\mathrm{f}_{6}\right)$ hold. Then for any positive integer $k$, there exists $\lambda_{0}>0$ such that for all $\lambda \geq \lambda_{0}$, the problem (1.1) has $k$ solutions $\left\{u_{j}\right\} \in W$ whose energy $I\left(u_{j}\right)<0$ for all $j=1, \ldots, k$.

This paper is organized as follows. In Section 2, we give some preliminary results of fractional Sobolev space with variable exponent. Furthermore, some compact embeddings are proved. In Section 3, by using Mountain Pass Theorem, we obtain the existence of weak solution for above problem. In Section 4, using Fountain Theorem, we get the existence of infinitely weak solutions for above problem. In Section 5 , we study the existence of $k$ weak solutions to problem (1.1) with negative energy via Genus theory.

\section{Some preliminary results}

Let $D$ be an open subset of $\mathbb{R}^{N}$, set

$$
L_{+}^{\infty}(D)=\left\{\mathfrak{p} \in L^{\infty}(D): \underset{x \in D}{\operatorname{essinf}} \mathfrak{p}(x) \geq 1\right\} .
$$

For $\mathfrak{p} \in L_{+}^{\infty}(D)$, let $\mathfrak{p}^{-}=\operatorname{essinf}_{x \in D} \mathfrak{p}(x), \mathfrak{p}^{+}=\operatorname{esssup}_{x \in D} \mathfrak{p}(x)$. Denote by $S(D)$ the set of all measurable real functions defined on $D$, elements in $S(D)$ which are equal to each other almost everywhere are considered as one element. For $\mathfrak{p} \in L_{+}^{\infty}(D)$, define

$$
L^{\mathfrak{p}(x)}(D)=\left\{u \in S(D): \int_{D}|u|^{\mathfrak{p}(x)} d x<+\infty\right\}
$$

with the norm

$$
\|u\|_{L^{\mathfrak{p}(x)}(D)}=\inf \left\{\lambda>0: \int_{D}\left|\frac{u}{\lambda}\right|^{\mathfrak{p}(x)} d x \leq 1\right\} .
$$

Lemma 2.1. 9 Set $\rho(u)=\int_{D}|u|^{\mathfrak{p}(x)} d x$. Assume that $u$ belongs to $L^{\mathfrak{p}(x)}(D)$, we have

(i) If $\|u\|_{L^{\mathfrak{p}(x)}(D)}>1$, then $\|u\|_{L^{\mathfrak{p}(x)}(D)}^{\mathfrak{p}^{-}} \leq \rho(u) \leq\|u\|_{L^{\mathfrak{p}(x)}(D)}^{\mathfrak{p}^{+}}$.

(ii) If $\|u\|_{L^{\mathfrak{p}(x)}(D)}<1$, then $\|u\|_{L^{\mathfrak{p}(x)}(D)}^{\mathfrak{p}^{+}} \leq \rho(u) \leq\|u\|_{L^{\mathfrak{p}(x)}(D)}^{\mathfrak{p}^{-}}$. 
From Lemma 2.1, we have

$$
\min \left\{\|u\|_{L^{\mathfrak{p}(x)}(D)}^{\mathfrak{p}^{+}},\|u\|_{L^{\mathfrak{p}(x)}(D)}^{\mathfrak{p}^{-}}\right\} \leq \rho(u) \leq \max \left\{\|u\|_{L^{\mathfrak{p}(x)}(D)}^{\mathfrak{p}^{+}},\|u\|_{L^{\mathfrak{p}(x)}(D)}^{\mathfrak{p}^{-}}\right\} .
$$

Lemma 2.2. $4,8,9$ The conjugate space of $L^{\mathfrak{p}(x)}(D)$ is $L^{\mathfrak{q}(x)}(D)$, where $\frac{1}{\mathfrak{p}(x)}+\frac{1}{\mathfrak{q}(x)}=1$. For any $u \in L^{\mathfrak{p}(x)}(D)$ and $v \in L^{\mathfrak{q}(x)}(D)$, we have

$$
\int_{D}|u v| d x \leq\left(\frac{1}{\mathfrak{p}^{-}}+\frac{1}{\mathfrak{q}^{-}}\right)\|u\|_{L^{\mathfrak{p}(x)}(D)}\|v\|_{L^{\mathfrak{q}(x)}(D)} \leq 2\|u\|_{L^{\mathfrak{p}(x)}(D)}\|v\|_{L^{\mathfrak{q}(x)}(D)} .
$$

Furthermore, if $\mathfrak{p}_{i} \in L_{+}^{\infty}(D), i=1, \ldots, k, k \geq 2$ and

$$
\frac{1}{\mathfrak{p}_{1}(x)}+\cdots+\frac{1}{\mathfrak{p}_{k}(x)}=1
$$

then for all $u_{i} \in L^{\mathfrak{p}_{i}(x)}(D), i=1, \ldots, k$, we have

$$
\begin{aligned}
\int_{D}\left|u_{1} \cdots u_{k}\right| d x & \leq\left(\frac{1}{\mathfrak{p}_{1}^{-}}+\cdots+\frac{1}{\mathfrak{p}_{k}^{-}}\right)\left\|u_{1}\right\|_{L^{\mathfrak{p}_{1}(x)}(D)} \cdots\left\|u_{k}\right\|_{L^{\mathfrak{p}_{k}(x)}(D)} \\
& \leq k\left\|u_{1}\right\|_{L^{\mathfrak{p}_{1}(x)}(D)} \cdots\|u\|_{L^{\mathfrak{p}_{k}(x)}(D)} .
\end{aligned}
$$

Let $\Omega$ be a Lipschitz domain in $\mathbb{R}^{N}$, we denote

$$
C_{+}(\Omega)=\left\{h \in C(\bar{\Omega}): 1<\inf _{x \in \bar{\Omega}} h(x) \leq \sup _{x \in \bar{\Omega}} h(x)<+\infty\right\} .
$$

Let $s: \bar{\Omega} \times \bar{\Omega} \rightarrow(0,1)$ be a continuous function satisfying the conditions $\left(\mathrm{S}_{1}\right)$ and $\left(\mathrm{S}_{2}\right)$ on $\bar{\Omega}$ and $p: \bar{\Omega} \times \bar{\Omega} \rightarrow(1,+\infty)$ be a continuous function satisfying the conditions:

$\left(\mathrm{P}_{1}\right) 1<p^{-} \leq p^{+}<+\infty$

$\left(\mathrm{P}_{2}\right) p$ is a symmetric function, that is, $p(x, y)=p(y, x)$ for all $(x, y) \in \bar{\Omega} \times \bar{\Omega}$.

For $q \in C_{+}(\Omega)$, define

$$
W^{s(\cdot), q(\cdot), p(\cdot)}(\Omega)=\left\{u \in L^{q(x)}(\Omega): \int_{\Omega \times \Omega} \frac{|u(x)-u(y)|^{p(x, y)}}{\lambda^{p(x, y)}|x-y|^{N+p(x, y) s(x, y)}} d x d y<+\infty\right\}
$$

for some $\lambda>0$. For $u \in W^{s(\cdot), q(\cdot), p(\cdot)}(\Omega)$, set

$$
[u]_{s(\cdot), p(\cdot), \Omega}=\inf \left\{\lambda>0: \int_{\Omega \times \Omega} \frac{|u(x)-u(y)|^{p(x, y)}}{\lambda^{p(x, y)}|x-y|^{N+p(x, y) s(x, y)}} d x d y \leq 1\right\} .
$$

Then $W^{s(\cdot), q(\cdot), p(\cdot)}(\Omega)$ is a Banach space with norm

$$
\|u\|_{W^{s(\cdot), q(\cdot), p(\cdot)}(\Omega)}=\|u\|_{L^{q(x)}(\Omega)}+[u]_{s(\cdot), p(\cdot), \Omega}
$$


We also consider another norm on $W^{s(\cdot), q(\cdot), p(\cdot)}(\Omega)$ as follows:

$$
\begin{aligned}
& \|u\| \|_{W^{s(\cdot), q(\cdot), p(\cdot)}(\Omega)} \\
= & \inf \left\{\lambda>0: \int_{\Omega \times \Omega} \frac{|u(x)-u(y)|^{p(x, y)}}{\lambda^{p(x, y)}|x-y|^{N+p(x, y) s(x, y)}} d x d y+\int_{\Omega} \frac{|u|^{q(x)}}{\lambda^{q(x)}} d x \leq 1\right\} .
\end{aligned}
$$

For any $\lambda>0$ such that

$$
\int_{\Omega \times \Omega} \frac{|u(x)-u(y)|^{p(x, y)}}{\lambda^{p(x, y)}|x-y|^{N+p(x, y) s(x, y)}} d x d y+\int_{\Omega} \frac{|u|^{q(x)}}{\lambda^{q(x)}} d x \leq 1,
$$

we obtain $\int_{\Omega \times \Omega} \frac{|u(x)-u(y)|^{p(x, y)}}{\lambda^{p(x, y)}|x-y|^{N+p(x, y) s(x, y)}} d x d y \leq 1$ and $\int_{\Omega} \frac{\mid u q^{q(x)}}{\lambda^{q(x)}} d x \leq 1$. Hence, we get $\lambda \in$ $\left\{\delta: \int_{\Omega \times \Omega} \frac{|u(x)-u(y)|^{p(x, y)}}{\delta^{p(x, y)}|x-y|^{N+p(x, y) s(x, y)}} d x d y \leq 1\right\}$ and $\lambda \in\left\{\beta: \int_{\Omega} \frac{|u|^{q(x)}}{\beta^{q(x)}} d x \leq 1\right\}$. We deduce

$$
\begin{aligned}
& {[u]_{s(\cdot), p(\cdot), \Omega}+\|u\|_{L^{q(x)}(\Omega)}} \\
& =\inf \left\{\delta: \int_{\Omega \times \Omega} \frac{|u(x)-u(y)|^{p(x, y)}}{\delta^{p(x, y)}|x-y|^{N+p(x, y) s(x, y)}} d x d y \leq 1\right\}+\inf \left\{\beta: \int_{\Omega} \frac{|u|^{q(x)}}{\beta^{q(x)}} d x \leq 1\right\} \\
& \leq 2 \inf \left\{\lambda>0: \int_{\Omega \times \Omega} \frac{|u(x)-u(y)|^{p(x, y)}}{\lambda^{p(x, y)}|x-y|^{N+p(x, y) s(x, y)}} d x d y+\int_{\Omega} \frac{|u|^{q(x)}}{\lambda^{q(x)}} d x \leq 1\right\} \\
& =2\|\| u \|_{W^{s(\cdot), q(\cdot), p(\cdot)}(\Omega)} .
\end{aligned}
$$

Furthermore, we see that

$$
\begin{aligned}
& \int_{\Omega \times \Omega} \frac{|u(x)-u(y)|^{p(x, y)}}{\left(2\|u\|_{W^{s(\cdot), q(\cdot), p(\cdot)}(\Omega)}\right)^{p(x, y)}|x-y|^{N+p(x, y) s(x, y)}} d x d y \\
+ & \int_{\Omega} \frac{|u|^{q(x)}}{\left(2\|u\|_{W^{s(\cdot), q(\cdot), p(\cdot)}(\Omega)}\right)^{q(x)}} d x \leq 1 .
\end{aligned}
$$

Therefore we get \|\|$u\left\|_{W^{s(\cdot), q(\cdot), p(\cdot)}(\Omega)} \leq 2\right\| u \|_{W^{s(\cdot), q(\cdot), p(\cdot)}(\Omega)}$. This means that two that norms are equivalent on $W^{s(\cdot), q(\cdot), p(\cdot)}(\Omega)$. The space $W^{s(\cdot), q(\cdot), p(\cdot)}\left(\mathbb{R}^{N}\right)$ is defined similarly as above, and it is a Banach space with norm

$$
\|u\|_{W^{s(\cdot), q(\cdot), p(\cdot)\left(\mathbb{R}^{N}\right)}}=\|u\|_{L^{q(x)}\left(\mathbb{R}^{N}\right)}+[u]_{s(\cdot), p(\cdot)},
$$

where

$$
[u]_{s(\cdot), p(\cdot)}=\inf \left\{\lambda>0: \int_{\mathbb{R}^{N} \times \mathbb{R}^{N}} \frac{|u(x)-u(y)|^{p(x, y)}}{\lambda^{p(x, y)}|x-y|^{N+p(x, y) s(x, y)}} d x d y \leq 1\right\} .
$$

When $q(x)=p(x, x)$, we write $W^{s(\cdot), p(\cdot)}\left(\mathbb{R}^{N}\right)$ instead of $W^{s(\cdot), q(\cdot), p(\cdot)}\left(\mathbb{R}^{N}\right)$.

Note that we just know $[\cdot]_{s(\cdot), p(\cdot)}$ is seminorm on $D^{s(\cdot), q(\cdot), p(\cdot)}\left(\mathbb{R}^{N}\right)$, where $D^{s(\cdot), q(\cdot), p(\cdot)}\left(\mathbb{R}^{N}\right)$ is the linear space of Lebesgue measurable functions on $\mathbb{R}^{N}$ such that

$$
\int_{\mathbb{R}^{N} \times \mathbb{R}^{N}} \frac{|u(x)-u(y)|^{p(x, y)}}{\lambda^{p(x, y)}|x-y|^{N+p(x, y) s(x, y)}} d x d y<+\infty
$$


for some $\lambda>0$. Then if we can show this seminorm is norm and there exists an embedding continuous from $L^{r(x)}\left(\mathbb{R}^{N}\right)$ into $D^{s(\cdot), q(\cdot), p(\cdot)}\left(\mathbb{R}^{N}\right)$ for some $r \in C_{+}\left(\mathbb{R}^{N}\right)$, then $D^{s(\cdot), q(\cdot), p(\cdot)}\left(\mathbb{R}^{N}\right)$ is a Banach space. Furthermore, it is a uniformly convex space, and this implies that $W^{s(\cdot), q(\cdot), p(\cdot)}\left(\mathbb{R}^{N}\right)$ is a reflexive Banach space. This problem explain why our work just focus on $H^{s(\cdot)}\left(\mathbb{R}^{N}\right)$. If that property is proved, then we believe that our work can extend for the problem

$$
(-\Delta)_{p(\cdot)}^{s(\cdot)} u+V(x)|u|^{p(x, x)-2} u=\lambda f(x, u) \quad \text { in } \mathbb{R}^{N},
$$

or Kirchhoff-type problems with related forms, where $(-\Delta)_{p(\cdot)}^{s(\cdot)}$ is defined as follows: for each $x \in \mathbb{R}^{N}$,

$$
(-\Delta)_{p(\cdot)}^{s(\cdot)} \varphi(x)=2 \mathrm{P} . \mathrm{V} \cdot \int_{\mathbb{R}^{N}} \frac{|\varphi(x)-\varphi(y)|^{p(x, y)-2}(\varphi(x)-\varphi(y))}{|x-y|^{N+p(x, y) s(x, y)}} d y
$$

along any $\varphi \in C_{0}^{\infty}\left(\mathbb{R}^{N}\right)$.

By arguments as in [10, Lemma 3.2], we get the result as follows:

Lemma 2.3. Let $\Omega$ be a Lipschitz domain, and $s, p, q$ as above. Assume furthermore that $N>s^{+} p^{+}$and $q(x) \geq p(x, x)$ for all $x \in \bar{\Omega}$. Then the embedding from $W^{s(\cdot), q(\cdot), p(\cdot)}(\Omega)$ into $L^{r(x)}(\Omega)$ is compact for any $r \in C_{+}(\Omega)$ such that $r(x)<p_{s}^{*}(x)=\frac{N p(x, x)}{N-p(x, x) s(x, x)}$ for all $x \in \bar{\Omega}$.

By using the method in the proof of Theorem 3.5 [10] and using Lemma 2.3, we get the following result.

Lemma 2.4. Let $s, p$ be uniformly continuous functions on $\mathbb{R}^{N} \times \mathbb{R}^{N}$ as above and $N>s^{+} p^{+}$. Then $W^{s(\cdot), p(\cdot)}\left(\mathbb{R}^{N}\right) \hookrightarrow L^{r(x)}\left(\mathbb{R}^{N}\right)$ and $W^{s(\cdot), p(\cdot)}\left(\mathbb{R}^{N}\right) \hookrightarrow \hookrightarrow L_{\mathrm{loc}}^{r(x)}\left(\mathbb{R}^{N}\right)$ for any uniformly continuous function $r \in C_{+}\left(\mathbb{R}^{N}\right)$ such that $r(x) \geq p(x, x)$ for all $x \in \mathbb{R}^{N}$ and $\inf _{x \in \mathbb{R}^{N}}\left(p_{s}^{*}(x)-r(x)\right)>0$.

Let $V$ satisfy $\left(\mathrm{V}_{1}\right)$, we denote $W_{V}^{s(\cdot), p(\cdot)}\left(\mathbb{R}^{N}\right)$ by the completion of $C_{0}^{\infty}\left(\mathbb{R}^{N}\right)$ with norm

$$
\|u\|_{W_{V}^{s(\cdot), p(\cdot)}\left(\mathbb{R}^{N}\right)}=\inf \left\{\lambda>0: \widetilde{\rho}_{V}\left(\frac{u}{\lambda}\right) \leq 1\right\}
$$

for $u \in W_{V}^{s(\cdot), p(\cdot)}\left(\mathbb{R}^{N}\right)$, where

$$
\widetilde{\rho}_{V}(u)=\int_{\mathbb{R}^{2 N}} \frac{|u(x)-u(y)|^{p(x, y)}}{|x-y|^{N+p(x, y) s(x, y)}} d x d y+\int_{\mathbb{R}^{N}} V(x)|u|^{p(x, x)} d x .
$$

When $p(x, y)=2$, we get $W_{V}^{s(\cdot), 2}\left(\mathbb{R}^{N}\right) \equiv W$. From Lemma 2.4, we get the following result. 
Lemma 2.5. Suppose that $V$ satisfies $\left(\mathrm{V}_{1}\right)$ and $\left(\mathrm{V}_{2}\right)$, s satisfies $\left(\mathrm{S}_{1}\right)$ and $\left(\mathrm{S}_{2}\right)$ on $\mathbb{R}^{N} \times$ $\mathbb{R}^{N}$, and p satisfies the condition $\left(\mathrm{P}_{1}\right)$ and $\left(\mathrm{P}_{2}\right)$ on $\mathbb{R}^{N} \times \mathbb{R}^{N}$, then $W_{V}^{s(\cdot), p(\cdot)}\left(\mathbb{R}^{N}\right)$ is embedded compactly into $L^{p(x, x)}\left(\mathbb{R}^{N}\right)$. Furthermore, $W_{V}^{s(\cdot), p(\cdot)}\left(\mathbb{R}^{N}\right)$ is also embedded compactly into $L^{q(x)}\left(\mathbb{R}^{N}\right)$ for any uniformly continuous function $q \in C_{+}\left(\mathbb{R}^{N}\right)$ such that $q(x)>p(x, x)$ for all $x \in \mathbb{R}^{N}$ and $\inf _{x \in \mathbb{R}^{N}}\left(p_{s}^{*}(x)-q(x)\right)>0$, where $p_{s}^{*}(x)=\frac{N p(x, x)}{N-p(x, x) s(x, x)}$.

Proof. From $\left(\mathrm{V}_{1}\right)$, for each $\lambda>0$, and all $u \in W_{V}^{s(\cdot), p(\cdot)}\left(\mathbb{R}^{N}\right)$, we have

$$
\begin{aligned}
& \min \left\{1, V^{-}\right\}\left(\int_{\mathbb{R}^{2 N}} \frac{|u(x)-u(y)|^{p(x, y)}}{\lambda^{p(x, y)}|x-y|^{N+p(x, y) s(x, y)}} d x d y+\int_{\mathbb{R}^{N}} \frac{|u|^{p(x, x)}}{\lambda^{p(x, x)}} d x\right) \\
\leq & \int_{\mathbb{R}^{2 N}} \frac{|u(x)-u(y)|^{p(x, y)}}{\lambda^{p(x, y)}|x-y|^{N+p(x, y) s(x, y)}} d x d y+\int_{\mathbb{R}^{N}} V(x) \frac{|u|^{p(x, x)}}{\lambda^{p(x, x)}} d x .
\end{aligned}
$$

Therefore,

$$
\int_{\mathbb{R}^{2 N}} \frac{|u(x)-u(y)|^{p(x, y)}}{\lambda^{p(x, y)}|x-y|^{N+p(x, y) s(x, y)}} d x d y+\int_{\mathbb{R}^{N}} V(x) \frac{|u|^{p(x, x)}}{\lambda^{p(x, x)}} d x \leq 1
$$

implies that

$$
\begin{aligned}
& \int_{\mathbb{R}^{2 N}} \frac{|u(x)-u(y)|^{p(x, y)}}{\left(\lambda \min \left\{1, V^{-}\right\}^{-1 / p^{+}}\right)^{p(x, y)}|x-y|^{N+p(x, y) s(x, y)}} d x d y \\
+ & \int_{\mathbb{R}^{N}} \frac{|u|^{p(x, x)}}{\left(\lambda \min \left\{1, V^{-}\right\}^{-1 / p^{+}}\right)^{p(x, x)}} d x \leq 1 .
\end{aligned}
$$

Hence for all $\lambda>0, \widetilde{\rho}_{V}\left(\frac{u}{\lambda}\right) \leq 1$, we get $\min \left\{1, V^{-}\right\}^{1 / p^{+}} \lambda$ belongs to the set $\{\beta>0$ : $\left.\widetilde{\rho}\left(\frac{u}{\beta}\right) \leq 1\right\}$, where

$$
\widetilde{\rho}(u)=\int_{\mathbb{R}^{2 N}} \frac{|u(x)-u(y)|^{p(x, y)}}{|x-y|^{N+p(x, y) s(x, y)}} d x d y+\int_{\mathbb{R}^{N}}|u|^{p(x, x)} d x .
$$

Then, we deduce

$$
\begin{aligned}
\|u\|_{W^{s(\cdot), p(\cdot)\left(\mathbb{R}^{N}\right)}} & =\inf \left\{\beta>0: \widetilde{\rho}\left(\frac{u}{\beta}\right) \leq 1\right\} \\
& \leq \min \left\{1, V^{-}\right\}^{-1 / p^{+}} \inf \left\{\lambda>0: \widetilde{\rho}_{V}\left(\frac{u}{\lambda}\right) \leq 1\right\} \\
& =\min \left\{1, V^{-}\right\}^{-1 / p^{+}}\|u\|_{W_{V}^{s(\cdot), p(\cdot)}\left(\mathbb{R}^{N}\right)}
\end{aligned}
$$

Then $W_{V}^{s(\cdot), p(\cdot)}\left(\mathbb{R}^{N}\right)$ is embedded continuously into $W^{s(\cdot), p(\cdot)}\left(\mathbb{R}^{N}\right)$. Using Lemma 2.4 . we get that $W_{V}^{s(\cdot), p(\cdot)}\left(\mathbb{R}^{N}\right)$ is embedded continuously into $L^{q(x)}\left(\mathbb{R}^{N}\right)$ for any uniformly continuous function $q \in C_{+}\left(\mathbb{R}^{N}\right)$ such that $p(x, x) \leq q(x)<p_{s}^{*}(x)$ for all $x \in \mathbb{R}^{N}$, $\inf _{x \in \mathbb{R}^{N}}\left(p_{s}^{*}(x)-q(x)\right)>0$, and this embedding is locally compactness. First, we prove that 
$W_{V}^{s(\cdot), p(\cdot)}\left(\mathbb{R}^{N}\right)$ is embedded compactly into $L^{p(x, x)}\left(\mathbb{R}^{N}\right)$. Let $u_{n} \rightarrow 0$ weak in $W_{V}^{s(\cdot), p(\cdot)}\left(\mathbb{R}^{N}\right)$, we prove $u_{n} \rightarrow 0$ strongly $L^{p(x, x)}\left(\mathbb{R}^{N}\right)$, and it is equivalent with $\lim _{n \rightarrow+\infty} \rho_{p}\left(\lambda u_{n}\right)=0$ for any fixed $\lambda>0$ by Lemma 2.1.9 in 8, where $\rho_{p}(u)=\int_{\mathbb{R}^{N}}|u|^{p(x, x)} d x$. By Lemma 2.4, we have compact embedding from $W_{V}^{s(\cdot), p(\cdot)}\left(\mathbb{R}^{N}\right) \hookrightarrow L_{\text {loc }}^{p(x, x)}\left(\mathbb{R}^{N}\right)$. It is enough to show that there exists $R>0$ such that

$$
\int_{\mathbb{R}^{N} \backslash B_{R}}\left|\lambda u_{n}\right|^{p(x, x)} d x \leq \varepsilon
$$

for any $\varepsilon>0$, and any fixed $\lambda>0$ and $n=1,2, \ldots$, where $B_{R}=\left\{x \in \mathbb{R}^{N}:|x|<R\right\}$. Set

$$
A(R, c)=\left\{x \in \mathbb{R}^{N} \backslash B_{R}: V(x) \geq c\right\} \quad \text { and } \quad B(R, c)=\left\{x \in \mathbb{R}^{N} \backslash B_{R}: V(x)<c\right\},
$$

where $c>0$. We see

$$
\int_{A(R, c)}\left|\lambda u_{n}(x)\right|^{p(x, x)} d x \leq \int_{\mathbb{R}^{N}} \frac{V(x)}{c}\left|\lambda u_{n}(x)\right|^{p(x, x)} d x \leq \frac{\left(\lambda^{p^{+}}+\lambda^{p^{-}}\right) \rho_{p, V}\left(u_{n}\right)}{c},
$$

where $\rho_{p, V}(u)=\int_{\mathbb{R}^{N}} V(x)|u|^{p(x, x)} d x$. We have

$$
\begin{aligned}
\rho_{p, V}\left(u_{n}\right) & \leq\left\|u_{n}\right\|_{L_{V}^{p(x, x)}\left(\mathbb{R}^{N}\right)}^{p^{+}}+\left\|u_{n}\right\|_{L_{V}^{p(x, x)}\left(\mathbb{R}^{N}\right)}^{p^{-}} \\
& \leq\left\|u_{n}\right\|_{W_{V}^{s(\cdot), p(\cdot)}\left(\mathbb{R}^{N}\right)}^{p^{+}}+\left\|u_{n}\right\|_{W_{V}^{s(\cdot), p(\cdot)}\left(\mathbb{R}^{N}\right)}^{p^{-}}<+\infty
\end{aligned}
$$

for all $n$ since $\left\{u_{n}\right\}$ is a bounded sequence in $W_{V}^{s(\cdot), p(\cdot)}\left(\mathbb{R}^{N}\right)$.

Choose $\sigma \in\left(1, \frac{N-\varepsilon_{0}}{N-p^{-} s^{-}}\right), p^{-} s^{-}>\varepsilon_{0}>0$ and $\sigma^{\prime}>0$, where $\sigma^{\prime}>0$ such that $\frac{1}{\sigma}+\frac{1}{\sigma^{\prime}}=1$. We have $p(x, x)<\sigma p(x, x)<\frac{\left(N-\varepsilon_{0}\right) p(x, x)}{N-p(x, x) s(x, x)}$, and $\frac{\left(N-\varepsilon_{0}\right) p(x, x)}{N-p(x, x) s(x, x)}$ is a uniformly continuous on $\mathbb{R}^{N}$, and satisfying property

$$
\begin{aligned}
\inf _{x \in \mathbb{R}^{N}}\left(p_{s}^{*}(x)-\sigma p(x, x)\right) & \geq \inf _{x \in \mathbb{R}^{N}}\left(p_{s}^{*}(x)-\frac{\left(N-\varepsilon_{0}\right) p(x, x)}{N-p(x, x) s(x, x)}\right) \\
& =\inf _{x \in \mathbb{R}^{N}} \frac{\varepsilon_{0} p(x, x)}{N-p(x, x) s(x, x)} \\
& >\frac{\varepsilon_{0} p^{-}}{N-p^{-} s^{-}}>0 .
\end{aligned}
$$

By Lemma 2.4 the embedding from $W_{V}^{s(\cdot), p(\cdot)}\left(\mathbb{R}^{N}\right) \hookrightarrow L^{\sigma p(x, x)}\left(\mathbb{R}^{N}\right)$ is continuous. Applying the Hölder inequality, we have

$$
\begin{aligned}
& \int_{B(R, c)}\left|\lambda u_{n}(x)\right|^{p(x, x)} d x \leq\left(\int_{B(R, c)}\left|\lambda u_{n}(x)\right|^{\sigma p(x, x)}\right)^{1 / \sigma}(\operatorname{meas}(B(R, c)))^{1 / \sigma^{\prime}} \\
\leq & \left(\lambda^{\sigma p^{+}}+\lambda^{\sigma p^{-}}\right)^{1 / \sigma}\left(\int_{\mathbb{R}^{N}}\left|u_{n}(x)\right|^{\sigma p(x, x)}\right)^{1 / \sigma}(\operatorname{meas}(B(R, c)))^{1 / \sigma^{\prime}}
\end{aligned}
$$




$$
\begin{aligned}
& \leq\left(\lambda^{\sigma p^{+}}+\lambda^{\sigma p^{-}}\right)^{1 / \sigma}\left(\left\|u_{n}\right\|_{L^{\sigma p(x, x)}\left(\mathbb{R}^{N}\right)}^{\sigma p^{+}}+\left\|u_{n}\right\|_{L^{\sigma p(x, x)}\left(\mathbb{R}^{N}\right)}^{\sigma p^{-}}\right)^{1 / \sigma}(\operatorname{meas}(B(R, c)))^{1 / \sigma^{\prime}} \\
& \leq C\left(\lambda^{\sigma p^{+}}+\lambda^{\sigma p^{-}}\right)^{1 / \sigma}\left(\left\|u_{n}\right\|_{W_{V}^{s(\cdot), p(\cdot)}\left(\mathbb{R}^{N}\right)}^{\sigma p^{+}}+\left\|u_{n}\right\|_{W_{V}^{s(\cdot), p(\cdot)}\left(\mathbb{R}^{N}\right)}^{\sigma p^{-}}\right)^{1 / \sigma}(\operatorname{meas}(B(R, c)))^{1 / \sigma^{\prime}},
\end{aligned}
$$

where $C>0$ is a suitable constant. Here, we use the continuous embedding $W_{V}^{s(\cdot), p(\cdot)}\left(\mathbb{R}^{N}\right)$ $\rightarrow L^{\sigma p(x, x)}\left(\mathbb{R}^{N}\right)$. Since $\left\|u_{n}\right\|_{W}$ is bounded and $\left(\mathrm{V}_{2}\right)$ holds, then we may choose $R, c$ large enough such that $\left(\left\|u_{n}\right\|_{W_{V}^{s(\cdot), p(\cdot)}\left(\mathbb{R}^{N}\right)}^{p^{+}}+\left\|u_{n}\right\|_{W_{V}^{s(\cdot), p(\cdot)}\left(\mathbb{R}^{N}\right)}^{p^{-}}\right) / c$ and meas $(B(R, c))$ are small enough. Therefore, for all $\varepsilon>0$, from 2.2 ) and $(2.3)$, we get

$$
\int_{\mathbb{R}^{N} \backslash B_{R}}\left|\lambda u_{n}(x)\right|^{p(x, x)} d x=\int_{A(R, c)}\left|\lambda u_{n}(x)\right|^{p(x, x)} d x+\int_{B(R, c)}\left|\lambda u_{n}(x)\right|^{p(x, x)} d x<\varepsilon
$$

for any fixed $\lambda>0$, which implies $(2.1)$ is proved.

For any uniformly continuous function $q \in C_{+}\left(\mathbb{R}^{N}\right)$ such that $\inf _{x \in \mathbb{R}^{N}}\left(p_{s}^{*}(x)-q(x)\right)=$ $a_{0}>0$, then $q(x) \in\left(p(x, x), p_{s}^{*}(x)-\frac{a_{0}}{2}\right)$ for all $x \in \mathbb{R}^{N}$, there exists $\sigma(x) \in(0,1)$ such that $\frac{1}{q(x)}=\frac{\sigma(x)}{p(x, x)}+\frac{1-\sigma(x)}{p_{s}^{*}(x)-a_{0} / 2}$. Then we have

$$
1=\frac{1}{p(x, x) /(\sigma(x) q(x))}+\frac{1}{\left(p_{s}^{*}(x)-a_{0} / 2\right) /((1-\sigma(x)) q(x))},
$$

and $q(x)=\sigma(x) q(x)+(1-\sigma(x)) q(x)$. In order to prove $u_{n} \rightarrow 0$ in $L^{q(x)}\left(\mathbb{R}^{N}\right)$, by Lemma 2.1.9 in [8], it is enough to show that $\lim _{n \rightarrow+\infty} \rho\left(\lambda u_{n}\right)=0$ for all $\lambda>0$, where $\rho(u)=\int_{\mathbb{R}^{N}}|u|^{q(x)} d x$. By using Hölder inequality with variable exponent, we get

$$
\begin{aligned}
& \int_{\mathbb{R}^{N}}\left|\lambda u_{n}(x)\right|^{q(x)} d x=\int_{\mathbb{R}^{N}}\left|\lambda u_{n}(x)\right|^{q(x) \sigma(x)}\left|\lambda u_{n}(x)\right|^{(1-\sigma(x)) q(x)} d x \\
& \leq 2\left\|\left|\lambda u_{n}\right|^{p(x, x)}\right\|_{L^{p(x, x) /(\sigma(x) q(x))}} \cdot\left\|\left|\lambda u_{n}\right|^{p_{s}^{*}(x)-a_{0} / 2}\right\|_{L^{\left(p_{s}^{*}(x)-a_{0} / 2\right) /((1-\sigma(x)) q(x))}} \\
& \leq 2\left(\left(\int_{\mathbb{R}^{N}}\left|\lambda u_{n}(x)\right|^{p(x, x)} d x\right)^{1 /(p /(\sigma q))^{+}}+\left(\int_{\mathbb{R}^{N}}\left|\lambda u_{n}(x)\right|^{p(x, x)} d x\right)^{1 /(p /(\sigma q))^{-}}\right) \\
& \times\left(\left(\int_{\mathbb{R}^{N}}\left|\lambda u_{n}(x)\right|^{p_{s}^{*}(x)-a_{0} / 2} d x\right)^{1 /\left(\left(p_{s}^{*}-a_{0} / 2\right) /(1-\sigma) q\right)^{+}}\right. \\
& \left.+\left(\int_{\mathbb{R}^{N}}\left|\lambda u_{n}(x)\right|^{p_{s}^{*}(x)-a_{0} / 2} d x\right)^{1 /\left(\left(p_{s}^{*}-a_{0} / 2\right) /(1-\sigma) q\right)^{-}}\right) .
\end{aligned}
$$

We note that

$$
\begin{gathered}
\lambda^{p_{s}^{*}(x)-a_{0} / 2} \leq \lambda^{\left(p_{s}^{*}-a_{0} / 2\right)^{+}}+\lambda^{\left(p_{s}^{*}-a_{0} / 2\right)^{-}}, \\
\int_{\mathbb{R}^{N}}\left|\lambda u_{n}(x)\right|^{p(x, x)} d x \rightarrow 0
\end{gathered}
$$

as $n \rightarrow \infty$. Since $p_{s}^{*}-a_{0} / 2$ is a uniformly continuous function in $\mathbb{R}^{N}$ and $\inf _{x \in \mathbb{R}^{N}}\left(p_{s}^{*}(x)-\right.$ $\left.\left(p_{s}^{*}(x)-a_{0} / 2\right)\right)=a_{0} / 2>0$, then $W_{V}^{s(\cdot), p(\cdot)}\left(\mathbb{R}^{N}\right) \hookrightarrow L^{p_{s}^{*}(x)-a_{0} / 2}\left(\mathbb{R}^{N}\right)$ is continuous. Combining (2.4) to 2.5), we get $\int_{\mathbb{R}^{N}}\left|\lambda u_{n}(x)\right|^{q(x)} d x \rightarrow 0$ for any fix $\lambda>0$ since $\int_{\mathbb{R}^{N}}\left|u_{n}(x)\right|^{p_{s}^{*}(x)-a_{0} / 2}$ 
$d x$ is a bounded sequence due to the continuous embedding from $W_{V}^{s(\cdot), p(\cdot)}\left(\mathbb{R}^{N}\right)$ into $L^{p_{s}^{*}(x)-a_{0} / 2}\left(\mathbb{R}^{N}\right)$. This implies that $u_{n} \rightarrow 0$ in $L^{q(x)}\left(\mathbb{R}^{N}\right)$.

Lemma 2.6. Suppose that $V$ satisfies $\left(\mathrm{V}_{1}\right)$ and $\left(\mathrm{V}_{2}\right)$, s satisfies $\left(\mathrm{S}_{1}\right)$ and $\left(\mathrm{S}_{2}\right)$, then $W$ is embedded compactly into $L^{2}\left(\mathbb{R}^{N}\right)$. Furthermore, $W$ is also embedded compactly into $L^{q(x)}\left(\mathbb{R}^{N}\right)$ for any uniformly continuous function $q \in C_{+}\left(\mathbb{R}^{N}\right)$ such that $q(x)>2$ for all $x \in \mathbb{R}^{N}$ and $\inf _{x \in \mathbb{R}^{N}}\left(2_{s}^{*}(x)-q(x)\right)>0$, where $2_{s}^{*}(x)=\frac{2 N}{N-2 s(x, x)}$.

Proof. In this lemma, we have $p(x, y)=2$ for all $(x, y) \in \mathbb{R}^{N} \times \mathbb{R}^{N}$ and then $H^{s(\cdot)}\left(\mathbb{R}^{N}\right)=$ $W^{s(\cdot), 2}\left(\mathbb{R}^{N}\right)$. Applying Lemma 2.5 for $p=2$, we get the statement of Lemma 2.6 .

Remark 2.7. Due to the technique problem, we do not have the embedding continuous from $H^{s(\cdot)}\left(\mathbb{R}^{N}\right)$ into $L^{2_{s}^{*}(x)}\left(\mathbb{R}^{N}\right)$, then it is very interesting to consider this case. Now, this question is still open.

Lemma 2.8. Suppose that $V$ satisfies $\left(\mathrm{V}_{1}\right)$ and $\left(\mathrm{V}_{3}\right)$, s satisfies $\left(\mathrm{S}_{1}\right)$ and $\left(\mathrm{S}_{2}\right)$ on $\mathbb{R}^{N} \times$ $\mathbb{R}^{N}$, and p satisfies the condition $\left(\mathrm{P}_{1}\right)$ and $\left(\mathrm{P}_{2}\right)$ on $\mathbb{R}^{N} \times \mathbb{R}^{N}$, then $W_{V}^{s(\cdot), p(\cdot)}\left(\mathbb{R}^{N}\right)$ is embedded compactly into $L^{p(x, x)}\left(\mathbb{R}^{N}\right)$. Furthermore, $W_{V}^{s(\cdot), p(\cdot)}\left(\mathbb{R}^{N}\right)$ is also embedded compactly into $L^{q(x)}\left(\mathbb{R}^{N}\right)$ for any uniformly continuous function $q \in C_{+}\left(\mathbb{R}^{N}\right)$ such that $q(x)>p(x, x)$ for all $x \in \mathbb{R}^{N}$ and $\inf _{x \in \mathbb{R}^{N}}\left(p_{s}^{*}(x)-q(x)\right)>0$, where $p_{s}^{*}(x)=\frac{N p(x, x)}{N-p(x, x) s(x, x)}$.

Proof. First, we prove that $W_{V}^{s(\cdot), p(\cdot)}\left(\mathbb{R}^{N}\right)$ is embedded compactly into $L^{p(x, x)}\left(\mathbb{R}^{N}\right)$. Let $u_{n} \rightarrow 0$ weak in $W_{V}^{s(\cdot), p(\cdot)}\left(\mathbb{R}^{N}\right)$, then there exists $\kappa>0$ such that

$$
\sup _{n}\left\|u_{n}\right\|_{W_{V}^{s(\cdot), p(\cdot)}\left(\mathbb{R}^{N}\right)} \leq \kappa
$$

We prove that $u_{n} \rightarrow 0$ strongly $L^{p(x, x)}\left(\mathbb{R}^{N}\right)$, and it is equivalent with $\lim _{n \rightarrow+\infty} \rho_{p}\left(\lambda u_{n}\right)=0$ for any fixed $\lambda>0$ by Lemma 2.1.9 in [8], where $\rho_{p}(u)=\int_{\mathbb{R}^{N}}|u|^{p(x, x)} d x$. By Lemma 2.4 we have a compact embedding from $W_{V}^{s(\cdot), p(\cdot)}\left(\mathbb{R}^{N}\right) \hookrightarrow L_{\text {loc }}^{p(x, x)}\left(\mathbb{R}^{N}\right)$. It is enough to show that there exists $R>0$ such that

$$
\int_{\mathbb{R}^{N} \backslash B_{R}}\left|\lambda u_{n}\right|^{p(x, x)} d x \leq \varepsilon
$$

for any $\varepsilon>0$, and any fixed $\lambda>0$ and $n=1,2, \ldots$, where $B_{R}=\left\{x \in \mathbb{R}^{N}:|x|<R\right\}$. First, we choose $\left\{y_{i}\right\} \subset \mathbb{R}^{N}$ such that $\mathbb{R}^{N}=\bigcup_{i=1}^{\infty} B_{r}\left(y_{i}\right)$ and each $x \in \mathbb{R}^{N}$ is covered by at most $N+1$ such balls. So we have

$$
\begin{aligned}
& \int_{\mathbb{R}^{N} \backslash B_{R}}\left|\lambda u_{n}\right|^{p(x, x)} d x \leq \sum_{\left|y_{j}\right| \geq R-r} \int_{B_{r}\left(y_{i}\right)}\left|\lambda u_{n}\right|^{p(x, x)} d x \\
= & \sum_{\left|y_{i}\right| \geq R-r}\left(\int_{B_{r}\left(y_{i}\right) \cap\left\{x \in \mathbb{R}^{N}: V(x)>c\right\}}\left|\lambda u_{n}\right|^{p(x, x)} d x+\int_{A_{c}\left(y_{i}\right)}\left|\lambda u_{n}\right|^{p(x, x)} d x\right)
\end{aligned}
$$




$$
\leq\left(\lambda^{p^{+}}+\lambda^{p^{-}}\right) \sum_{\left|y_{i}\right| \geq R-r}\left(\int_{B_{r}\left(y_{i}\right) \cap\left\{x \in \mathbb{R}^{N}: V(x)>c\right\}}\left|u_{n}\right|^{p(x, x)} d x+\int_{A_{c}\left(y_{i}\right)}\left|u_{n}\right|^{p(x, x)} d x\right),
$$

where $A_{c}\left(y_{i}\right)=\left\{x \in \mathbb{R}^{N}: V(x) \leq c\right\} \cap B_{r}\left(y_{i}\right)$. Note that

$$
\int_{B_{r}\left(y_{i}\right) \cap\left\{x \in \mathbb{R}^{N}: V(x)>c\right\}}\left|u_{n}\right|^{p(x, x)} d x \leq \frac{1}{c} \int_{B_{r}\left(y_{i}\right)} V(x)\left|u_{n}\right|^{p(x, x)} d x .
$$

Choose a number $\sigma \in\left(1, \frac{N-\varepsilon_{0}}{N-p^{-} s^{-}}\right), p^{-} s^{-}>\varepsilon_{0}>0$, then the embedding

$$
W_{V}^{s(\cdot), p(\cdot)}\left(\mathbb{R}^{N}\right) \hookrightarrow L^{\sigma p(x, x)}\left(\mathbb{R}^{N}\right)
$$

is continuous and there exists a constant $C>0$ such that

$$
\left\|u_{n}\right\|_{L^{\sigma p(x, x)}\left(\mathbb{R}^{N}\right)} \leq C\left\|u_{n}\right\|_{W_{V}^{s(\cdot), p(\cdot)}\left(\mathbb{R}^{N}\right)} \leq C \kappa .
$$

Using Hölder inequality, we get

$$
\begin{aligned}
\int_{A_{c}\left(y_{i}\right)}\left|u_{n}\right|^{p(x, x)} & \leq\left(\int_{A_{c}\left(y_{i}\right)}\left|u_{n}\right|^{\sigma p(x, x)} d x\right)^{1 / \sigma}\left(\int_{A_{c}\left(y_{i}\right)} 1^{\sigma /(\sigma-1)} d x\right)^{(\sigma-1) / \sigma} \\
& \leq\left(\int_{B_{r}\left(y_{i}\right)}\left|u_{n}\right|^{\sigma p(x, x)} d x\right)^{1 / \sigma}\left(\operatorname{meas}\left(A_{c}\left(y_{i}\right)\right)\right)^{(\sigma-1) / \sigma} .
\end{aligned}
$$

Therefore, we deduce

$$
\begin{aligned}
& \int_{\mathbb{R}^{N} \backslash B_{R}}\left|\lambda u_{n}\right|^{p(x, x)} d x \\
\leq & \left(\lambda^{p^{+}}+\lambda^{p^{-}}\right) \sum_{\left|y_{j}\right| \geq R-r}\left(\frac{1}{c} \int_{B_{r}\left(y_{i}\right)} V(x)\left|u_{n}\right|^{p(x, x)} d x\right. \\
& \left.+\left(\int_{B_{r}\left(y_{i}\right)}\left|u_{n}\right|^{\sigma p(x, x)} d x\right)^{1 / \sigma}\left(\operatorname{meas}\left(A_{c}\left(y_{i}\right)\right)\right)^{(\sigma-1) / \sigma}\right) \\
\leq & \left(\lambda^{p^{+}}+\lambda^{p^{-}}\right)\left(\frac{N+1}{c} \int_{\mathbb{R}^{N}} V(x)\left|u_{n}\right|^{p(x, x)} d x\right. \\
& \left.+(N+1)^{1 / \sigma}\left(\int_{\mathbb{R}^{N}}\left|u_{n}\right|^{\sigma p(x, x)} d x\right)^{1 / \sigma} \sup _{|y| \geq R-r}\left(\operatorname{meas}\left(A_{c}(y)\right)\right)^{(\sigma-1) / \sigma}\right) .
\end{aligned}
$$

There exists $\kappa_{*}>0$ such that

$$
\begin{aligned}
\int_{\mathbb{R}^{N}} V(x)\left|u_{n}\right|^{p(x, x)} d x & \leq\left\|u_{n}\right\|_{L_{V}^{p(x, x)}\left(\mathbb{R}^{N}\right)}^{p^{+}}+\left\|u_{n}\right\|_{L_{V}^{p(x, x)}\left(\mathbb{R}^{N}\right)}^{p^{-}} \\
& \leq\left\|u_{n}\right\|_{W_{V}^{+(\cdot), p(\cdot)}\left(\mathbb{R}^{N}\right)}^{p^{+}}+\left\|u_{n}\right\|_{W_{V}^{s(\cdot), p(\cdot)}\left(\mathbb{R}^{N}\right)}^{p^{-}}<\kappa_{*}
\end{aligned}
$$


for all $n$ since $\left\{u_{n}\right\}$ is a bounded sequence in $W_{V}^{s(\cdot), p(\cdot)}\left(\mathbb{R}^{N}\right)$. We also have

$$
\int_{\mathbb{R}^{N}}\left|u_{n}\right|^{\sigma p(x, x)} d x \leq\left\|u_{n}\right\|_{L^{\sigma p(x, x)}}^{\sigma p^{+}}+\left\|u_{n}\right\|_{L^{\sigma p(x, x)}}^{\sigma p^{-}} \leq(C \kappa)^{\sigma p^{+}}+(C \kappa)^{\sigma p^{-}} .
$$

Combining 2.7 to 2.8), taking $c$ and $R$ large enough, we get 2.6). We have completed the proof of Lemma 2.8 .

From Lemma 2.8, setting $p(x, y)=2$ for all $(x, y) \in \mathbb{R}^{N} \times \mathbb{R}^{N}$, we get the following result.

Lemma 2.9. Suppose that $V$ satisfies $\left(\mathrm{V}_{1}\right)$ and $\left(\mathrm{V}_{3}\right), s$ satisfies $\left(\mathrm{S}_{1}\right)$ and $\left(\mathrm{S}_{2}\right)$, then $W$ is embedded compactly into $L^{2}\left(\mathbb{R}^{N}\right)$. Furthermore, $W$ is also embedded compactly into $L^{q(x)}\left(\mathbb{R}^{N}\right)$ for any uniformly continuous function $q \in C_{+}\left(\mathbb{R}^{N}\right)$ such that $q(x)>2$ for all $x \in \mathbb{R}^{N}$ and $\inf _{x \in \mathbb{R}^{N}}\left(2_{s}^{*}(x)-q(x)\right)>0$, where $2_{s}^{*}(x)=\frac{2 N}{N-2 s(x, x)}$.

By Lemmas 2.6 and 2.9, we get the following result.

Corollary 2.10. Assume that $V$ satisfies the conditions $\left(\mathrm{V}_{1}\right)$ and $\left(\mathrm{V}_{2}\right)$ or $\left(\mathrm{V}_{1}\right)$ and $\left(\mathrm{V}_{3}\right)$, then the embeddings from $W$ into $L^{2}\left(\mathbb{R}^{N}\right)$ and $W$ into $L^{q(x)}\left(\mathbb{R}^{N}\right)$ are continuous which $q \in C_{+}\left(\mathbb{R}^{N}\right)$ is a uniformly continuous function such that $q(x)>2$ for all $x \in \mathbb{R}^{N}$ and $\inf _{x \in \mathbb{R}^{N}}\left(2_{s}^{*}(x)-q(x)\right)>0$, where $2_{s}^{*}(x)=\frac{2 N}{N-2 s(x, x)}$. Hence, there exists a best constants $S_{2}>0$ and $S_{q}>0$ as follows:

$$
S_{2}=\sup _{u \neq 0, u \in W} \frac{\|u\|_{L^{2}\left(\mathbb{R}^{N}\right)}}{\|u\|_{W}} \quad \text { and } \quad S_{q}=\sup _{u \neq 0, u \in W} \frac{\|u\|_{L^{q(x)}\left(\mathbb{R}^{N}\right)}}{\|u\|_{W}}
$$

respectively. This implies $\|u\|_{L^{2}\left(\mathbb{R}^{N}\right)} \leq S_{2}\|u\|_{W}$ and $\|u\|_{L^{q(x)}\left(\mathbb{R}^{N}\right)} \leq S_{q}\|u\|_{W}$ for all $u \in W$.

Lemma 2.11. Assume that $V$ satisfies the conditions $\left(\mathrm{V}_{1}\right)$ and $\left(\mathrm{V}_{2}\right)$ or $\left(\mathrm{V}_{1}\right)$ and $\left(\mathrm{V}_{3}\right)$, $f$ satisfies the condition $(\mathfrak{f})$. Set $\psi(u)=\int_{\mathbb{R}^{N}} F(x, u) d x, u \in W$, then $\psi \in C^{1}(W, \mathbb{R})$ and $\psi^{\prime}$ is a operator compact on $W$. Furthermore, we have

$$
\left\langle\psi^{\prime}(u), v\right\rangle=\int_{\mathbb{R}^{N}} f(x, u(x)) v(x) d x
$$

for all $v \in W$.

Proof. By the condition (f), we have

$$
|F(x, u)| \leq\left|h_{0}(x)\right||u|+\sum_{i=1}^{m}\left|h_{i}(x)\right||u|^{\gamma_{i}(x)}+g_{m+1}|u|^{2}+h_{m+1}|u|^{q(x)} .
$$

Then, using Hölder inequality with variable exponent, we have

$$
\begin{aligned}
\int_{\mathbb{R}^{N}}|F(x, u)| d x \leq & 2\left\|h_{0}\right\|_{L^{q(x) /(q(x)-1)}}\|u\|_{L^{q(x)}\left(\mathbb{R}^{N}\right)}+g_{m+1} \int_{\mathbb{R}^{N}}|u|^{2} d x \\
& +2 \sum_{i=1}^{m}\left\|h_{i}\right\|_{L^{q(x) /\left(q(x)-\gamma_{i}(x)\right)\left(\mathbb{R}^{N}\right)}}\left\||u|^{\gamma_{i}}\right\|_{L^{q(x) /\left(\gamma_{i}(x)\right)}}+h_{m+1} \int_{\mathbb{R}^{N}}|u|^{q(x)} d x .
\end{aligned}
$$


From Lemmas 2.6 and 2.9, there exists a constant $C>0$ such that

$$
\|u\|_{L^{q(x)}\left(\mathbb{R}^{N}\right)} \leq C\|u\|_{W} \quad \text { and } \quad\|u\|_{L^{2}\left(\mathbb{R}^{N}\right)} \leq C\|u\|_{W}
$$

for any $u \in W$. By Lemma 2.1, we have

$$
\int_{\mathbb{R}^{N}}|u|^{q(x)} d x \leq \max \left\{\|u\|_{L^{q(x)}\left(\mathbb{R}^{N}\right)}^{q^{+}},\|u\|_{L^{q(x)}\left(\mathbb{R}^{N}\right)}^{q^{-}}\right\}
$$

and

$$
\begin{aligned}
& \left\||u|^{\gamma_{i}}\right\|_{L^{q(x) /\left(\gamma_{i}(x)\right)}} \leq \max \left\{\left(\int_{\mathbb{R}^{N}}|u|^{q(x)} d x\right)^{1 /\left(q / \gamma_{i}\right)^{+}},\left(\int_{\mathbb{R}^{N}}|u|^{q(x)} d x\right)^{1 /\left(q / \gamma_{i}\right)^{-}}\right\} \\
& \leq \max \left\{\left(\max \left\{\|u\|_{L^{q(x)}\left(\mathbb{R}^{N}\right)}^{q^{+}},\|u\|_{L^{q(x)}\left(\mathbb{R}^{N}\right)}^{q^{-}}\right\}\right)^{1 /\left(q / \gamma_{i}\right)^{+}},\right. \\
& \left.\left(\max \left\{\|u\|_{L^{q(x)}\left(\mathbb{R}^{N}\right)}^{q^{+}},\|u\|_{L^{q(x)}\left(\mathbb{R}^{N}\right)}^{q^{-}}\right\}\right)^{1 /\left(q / \gamma_{i}\right)^{-}}\right\} .
\end{aligned}
$$

Combining (2.9) to 2.12, we get

$$
\begin{aligned}
\int_{\mathbb{R}^{N}}|F(x, u)| d x \leq & 2\left\|h_{0}\right\|_{L^{q(x) /(q(x)-1)}}\|u\|_{L^{q(x)}\left(\mathbb{R}^{N}\right)}+g_{m+1}\|u\|_{L^{2}\left(\mathbb{R}^{N}\right)}^{2} \\
+ & 2 \sum_{i=1}^{m}\left\|h_{i}\right\|_{L^{q(x) /\left(q(x)-\gamma_{i}(x)\right)}\left(\mathbb{R}^{N}\right)} \\
& \times \max \left\{\left(\max \left\{\|u\|_{L^{q(x)}\left(\mathbb{R}^{N}\right)}^{q^{+}},\|u\|_{L^{q(x)}\left(\mathbb{R}^{N}\right)}^{q^{-}}\right\}\right)^{1 /\left(q / \gamma_{i}\right)^{+}},\right. \\
& \left.\left(\max \left\{\|u\|_{L^{q(x)}\left(\mathbb{R}^{N}\right)}^{q^{+}},\|u\|_{L^{q(x)}\left(\mathbb{R}^{N}\right)}^{q^{-}}\right\}\right)^{1 /\left(q / \gamma_{i}\right)^{-}}\right\} \\
+ & h_{m+1} \max \left\{\|u\|_{L^{q(x)}\left(\mathbb{R}^{N}\right)}^{q^{+}},\|u\|_{L^{q(x)}\left(\mathbb{R}^{N}\right)}^{q^{-}}\right\} .
\end{aligned}
$$

This implies that $\int_{\mathbb{R}^{N}}|F(x, u)| d x<+\infty$, then $\psi$ is well-defined on $W$. For any $|s| \in[0,1]$ and $u, v \in W$, we have

$$
|u+s v|^{\gamma_{i}-1} \leq(|u|+|v|)^{\gamma_{i}-1} \text { and } \quad|u+s v|^{q-1} \leq(|u|+|v|)^{q-1}
$$

for all $i=1, \ldots, m$. By using Young inequality, there exists a constant $D>0$ such that

$$
|u+s v \| v| \leq D\left(|u|^{2}+|v|^{2}\right) .
$$

We see that

$$
\begin{aligned}
|f(x, u+s v) v| \leq & \left|h_{0}(x)\right||v|+\sum_{i=1}^{m}\left|h_{i}(x)\right|(|u|+|v|)^{\gamma_{i}-1} \\
& +D g_{m+1}\left(|u|^{2}+|v|^{2}\right)+h_{m+1}(|u|+|v|)^{q-1} .
\end{aligned}
$$

From 2.14 and using Hölder inequality, we obtain

$$
\int_{\mathbb{R}^{N}}\left(\left|h_{0}(x)\right||v|+\sum_{i=1}^{m}\left|h_{i}(x)\right|(|u|+|v|)^{\gamma_{i}-1}+D g_{m+1}\left(|u|^{2}+|v|^{2}\right)\right.
$$




$$
\begin{aligned}
& \left.+h_{m+1}(|u|+|v|)^{q(x)-1}\right) d x \\
\leq & \left\|h_{0}\right\|_{L^{q(x) /(q(x)-1)\left(\mathbb{R}^{N}\right)}}\|v\|_{L^{q(x)}\left(\mathbb{R}^{N}\right)} \\
& +2 \sum_{i=1}^{m}\left\|h_{i}\right\|_{L^{q(x) /\left(q(x)-\gamma_{i}(x)\right)}\left(\mathbb{R}^{N}\right)}\left\|(|u|+|v|)^{\gamma_{i}}\right\|_{L^{q(x) / \gamma_{i}(x)}\left(\mathbb{R}^{N}\right)} \\
& +D g_{m+1}\left(\|u\|_{L^{2}\left(\mathbb{R}^{N}\right)}^{2}+\|v\|_{L^{2}\left(\mathbb{R}^{N}\right)}^{2}\right) \\
+ & 2 h_{m+1}\left\|(|u|+|v|)^{q(x)-1}\right\|_{L^{q(x) /(q(x)-1)\left(\mathbb{R}^{N}\right)}\|u\|_{L^{q(x)}\left(\mathbb{R}^{N}\right)} .}
\end{aligned}
$$

Note that $(|u|+|v|)^{q(x)} \leq 2^{q(x)}\left(|u|^{q(x)}+|v|^{q(x)}\right) \leq 2^{q^{+}}\left(|u|^{q(x)}+|v|^{q(x)}\right)$, then we have

$$
\begin{aligned}
& \left\|(|u|+|v|)^{\gamma_{i}}\right\|_{L^{q(x) / \gamma_{i}(x)}\left(\mathbb{R}^{N}\right)} \\
\leq & \max \left\{\left(\int_{\mathbb{R}^{N}}(|u|+|v|)^{q(x)} d x\right)^{1 /\left(q / \gamma_{i}\right)^{+}},\left(\int_{\mathbb{R}^{N}}(|u|+|v|)^{q(x)} d x\right)^{1 /\left(q / \gamma_{i}\right)^{-}}\right\} \\
\leq & \max \left\{2^{q^{+} /\left(q / \gamma_{i}\right)^{+}}\left(\int_{\mathbb{R}^{N}}\left(|u|^{q(x)}+|v|^{q(x)}\right) d x\right)^{1 /\left(q / \gamma_{i}\right)^{+}},\right. \\
& \left.2^{q^{+} /\left(q / \gamma_{i}\right)^{-}}\left(\int_{\mathbb{R}^{N}}\left(|u|^{q(x)}+|v|^{q(x)}\right) d x\right)^{1 /\left(q / \gamma_{i}\right)^{-}}\right\}
\end{aligned}
$$

and

$$
\begin{aligned}
& \left\|(|u|+|v|)^{q(x)-1}\right\|_{L^{q(x) /(q(x)-1)}\left(\mathbb{R}^{N}\right)} \\
\leq & \max \left\{\left(\int_{\mathbb{R}^{N}}(|u|+|v|)^{q(x)} d x\right)^{1 /(q /(q-1))^{+}},\left(\int_{\mathbb{R}^{N}}(|u|+|v|)^{q(x)} d x\right)^{1 /(q /(q-1))^{-}}\right\} \\
\leq & \max \left\{2^{q^{+} /(q /(q-1))^{+}}\left(\int_{\mathbb{R}^{N}}\left(|u|^{q(x)}+|v|^{q(x)}\right) d x\right)^{1 /(q /(q-1))^{+}},\right. \\
& \left.2^{q^{+} /(q /(q-1))^{-}}\left(\int_{\mathbb{R}^{N}}\left(|u|^{q(x)}+|v|^{q(x)}\right) d x\right)^{1 /(q /(q-1))^{-}}\right\} .
\end{aligned}
$$

Combining 2.10 to 2.15 , we get

$\left|h_{0}(x)\right||v|+\sum_{i=1}^{m}\left|h_{i}(x)\right|(|u|+|v|)^{\gamma_{i}-1}+D g_{m+1}\left(|u|^{2}+|v|^{2}\right)+h_{m+1}(|u|+|v|)^{q-1} \in L^{1}\left(\mathbb{R}^{N}\right)$.

By the mean-value theorem and the Lebesgue dominated convergence theorem, there exists function $\theta$ with $\theta(x) \in[0,1]$ such that

$$
\begin{aligned}
\left\langle\psi^{\prime}(u), v\right\rangle & =\lim _{s \rightarrow 0} \frac{\psi(u+s v)-\psi(u)}{s}=\lim _{s \rightarrow 0} \int_{\mathbb{R}^{N}} \frac{F(x, u+s v)-F(x, u)}{s} d x \\
& =\lim _{s \rightarrow 0} \int_{\mathbb{R}^{N}} f(x, u+\theta s v) v d x=\int_{\mathbb{R}^{N}} f(x, u) v d x
\end{aligned}
$$


due to $|s \theta(x)| \in[0,1]$ for all $x \in \mathbb{R}^{N}$. This shows that $\psi$ is Gateaux differentiable. Applying the condition (f), we have

$$
\begin{aligned}
\left|\left\langle\psi^{\prime}(u), v\right\rangle\right| \leq & \int_{\mathbb{R}^{N}}|f(x, u) v| d x \\
\leq & \int_{\mathbb{R}^{N}}\left|h_{0}(x)\right||v| d x \\
& +\int_{\mathbb{R}^{N}}\left(\sum_{i=1}^{m}\left|h_{i}(x)\right||u|^{\gamma_{i}(x)-1}|v|+g_{m+1}|u||v|+h_{m+1}|u|^{q(x)-1}|v|\right) d x .
\end{aligned}
$$

Using Hölder inequality, we get

$$
\int_{\mathbb{R}^{N}}\left|h_{0}(x)\|v \mid d x \leq 2\| h_{0}\left\|_{L^{q(x) /(q(x)-1)\left(\mathbb{R}^{N}\right)}}\right\| v \|_{L^{q(x)}\left(\mathbb{R}^{N}\right)}\right.
$$

and

$$
\int_{\mathbb{R}^{N}}|u|^{q(x)-1}|v| d x \leq 2\left\||u|^{q(x)-1}\right\|_{L^{q(x) /(q(x)-1)}\left(\mathbb{R}^{N}\right)}\|v\|_{L^{q(x)}\left(\mathbb{R}^{N}\right)} .
$$

Note that

$$
\begin{aligned}
& \left\||u|^{q(x)-1}\right\|_{L^{q(x) /(q(x)-1)}\left(\mathbb{R}^{N}\right)} \\
\leq & \max \left\{\left(\int_{\mathbb{R}^{N}}|u|^{q(x)} d x\right)^{1 /(q /(q-1))^{+}},\left(\int_{\mathbb{R}^{N}}|u|^{q(x)} d x\right)^{1 /(q /(q-1))^{-}}\right\} \\
\leq & \max \left\{\left(\max \left\{\|u\|_{L^{q(x)}\left(\mathbb{R}^{N}\right)}^{q^{+}},\|u\|_{L^{q(x)}\left(\mathbb{R}^{N}\right)}^{q^{-}}\right\}\right)^{1 /(q /(q-1))^{+}},\right. \\
& \left.\left(\max \left\{\|u\|_{L^{q(x)}\left(\mathbb{R}^{N}\right)}^{q^{+}},\|u\|_{L^{q(x)}\left(\mathbb{R}^{N}\right)}^{q^{-}}\right\}\right)^{1 /(q /(q-1))^{-}}\right\} .
\end{aligned}
$$

Since $\frac{1}{q(x)}+\frac{\gamma_{i}-1}{q(x)}+\frac{q(x)-\gamma_{i}(x)}{q(x)}=1$, applying Lemma 2.2, we have

$$
\int_{\mathbb{R}^{N}}\left|h_{i}(x)\right||u|^{\gamma_{i}-1}|v| d x \leq 3\left\|h_{i}\right\|_{L^{q(x) /\left(q(x)-\gamma_{i}(x)\right)}\left(\mathbb{R}^{N}\right)}\left\||u|^{\gamma_{i}-1}\right\|_{L^{q(x) /\left(\gamma_{i}(x)-1\right)}\left(\mathbb{R}^{N}\right)}\|v\|_{L^{q(x)}\left(\mathbb{R}^{N}\right)}
$$

for all $i=1, \ldots, m$. Combining (2.10)-2.12, 2.16) to (2.17), we get

$$
\begin{aligned}
\left|\left\langle\psi^{\prime}(u), v\right\rangle\right| \leq & 2\left\|h_{0}\right\|_{L^{q(x) /(q(x)-1)}\left(\mathbb{R}^{N}\right)}\|v\|_{L^{q(x)}\left(\mathbb{R}^{N}\right)} \\
+ & 3 \sum_{i=1}^{m}\left\|h_{i}\right\|_{L^{q(x) /\left(q(x)-\gamma_{i}(x)\right)}\left(\mathbb{R}^{N}\right)} \\
& \times \max \left\{\left(\max \left\{\|u\|_{L^{q(x)}\left(\mathbb{R}^{N}\right)}^{q^{+}},\|u\|_{L^{q(x)}\left(\mathbb{R}^{N}\right)}^{q^{-}}\right\}\right)^{1 /\left(q / \gamma_{i}\right)^{+}},\right. \\
& \left.\quad\left(\max \left\{\|u\|_{L^{q(x)}\left(\mathbb{R}^{N}\right)}^{q^{+}},\|u\|_{L^{q(x)}\left(\mathbb{R}^{N}\right)}^{q^{-}}\right\}\right)^{1 /\left(q / \gamma_{i}\right)^{-}}\right\}\|v\|_{L^{q(x)}\left(\mathbb{R}^{N}\right)} \\
+ & g_{m+1}\|u\|_{L^{2}\left(\mathbb{R}^{N}\right)}\|v\|_{L^{2}\left(\mathbb{R}^{N}\right)} \\
+ & \max \left\{\left(\max \left\{\|u\|_{L^{q(x)}\left(\mathbb{R}^{N}\right)}^{q^{+}},\|u\|_{L^{q(x)}\left(\mathbb{R}^{N}\right)}^{q^{-}}\right\}\right)^{1 /(q /(q-1))^{+}},\right. \\
& \left.\left(\max \left\{\|u\|_{L^{q(x)}\left(\mathbb{R}^{N}\right)}^{q^{+}},\|u\|_{L^{q(x)}\left(\mathbb{R}^{N}\right)}^{q^{-}}\right\}\right)^{1 /(q /(q-1))^{-}}\right\}\|v\|_{L^{q(x)}\left(\mathbb{R}^{N}\right)} \\
\leq & C_{u}\|v\|_{W},
\end{aligned}
$$


where $C_{u}>0$ is a constant depending on the function $h_{0}, \ldots, h_{m}, u$. This implies that $\psi^{\prime}(u) \in W^{*}$.

We now prove that $\psi^{\prime}: W \rightarrow W^{*}$ is weakly continuous. Suppose that $u_{n} \rightarrow u$ in $W$. By Corollary 2.10, we can assume that $u_{n} \rightarrow u$ in $L^{q(x)}\left(\mathbb{R}^{n}\right)$ and $L^{2}\left(\mathbb{R}^{N}\right)$ and $u_{n}(x) \rightarrow u(x)$ for all $x \in \mathbb{R}^{N}$. We have

$$
\begin{aligned}
\left\|\psi^{\prime}\left(u_{n}\right)-\psi^{\prime}(u)\right\| & =\sup _{\|v\|_{W}=1}\left|\left\langle\psi^{\prime}\left(u_{n}\right)-\psi^{\prime}(u), v\right\rangle\right| \\
& =\sup _{\|v\|_{W}=1}\left|\int_{\mathbb{R}^{N}}\left(f\left(x, u_{n}\right) v-f(x, u) v\right) d x\right| .
\end{aligned}
$$

Set $\rho:=\lim _{n \rightarrow \infty} \sup _{\|v\|_{W}=1}\left|\int_{\mathbb{R}^{N}}\left(f\left(x, u_{n}\right) v-f(x, u) v\right) d x\right|$. It is sufficient to show that $\rho=0$. Conversely, if $\rho>0$, then there exists a sequence $\left\{v_{n}\right\} \subset W,\left\|v_{n}\right\|_{W}=1$ such that

$$
\left|\int_{\mathbb{R}^{N}}\left(f\left(x, u_{n}\right) v_{n}-f(x, u) v_{n}\right) d x\right|>\frac{\rho}{2}
$$

for all $n$ large enough. Since $\left\{v_{n}\right\}$ is bounded in $W$, by Corollary 2.10, we can assume that $v_{n} \rightarrow v$ in $L^{q(x)}\left(\mathbb{R}^{n}\right), L^{2}\left(\mathbb{R}^{N}\right)$ and $v_{n}(x) \rightarrow u(x)$ for all $x \in \mathbb{R}^{N}$. By argument as in (2.18), we have

$$
\begin{aligned}
& \left|\int_{\mathbb{R}^{N}}\left(f\left(x, u_{n}\right) v_{n}-f(x, u) v_{n}\right) d x\right| \leq \int_{\mathbb{R}^{N}}\left|f\left(x, u_{n}\right) v_{n}-f(x, u) v_{n}\right| d x \\
\leq & \int_{\mathbb{R}^{N}}\left(\left|f\left(x, u_{n}\right) v_{n}\right|+\left|f(x, u) v_{n}\right|\right) d x \leq 2\left\|h_{0}\right\|_{L^{q(x) /(q(x)-1)}\left(\mathbb{R}^{N}\right)}\left\|v_{n}\right\|_{L^{q(x)}\left(\mathbb{R}^{N}\right)} \\
+ & 3 \sum_{i=1}^{m}\left\|h_{i}\right\|_{L^{q(x) /\left(q(x)-\gamma_{i}(x)\right)}\left(\mathbb{R}^{N}\right)} \max \left\{\left(\max \left\{\left\|u_{n}\right\|_{L^{q(x)}\left(\mathbb{R}^{N}\right)}^{q^{+}},\left\|u_{n}\right\|_{L^{q(x)}\left(\mathbb{R}^{N}\right)}^{q^{-}}\right\}\right)^{1 /\left(q / \gamma_{i}\right)^{+}},\right. \\
& \left.\left(\max \left\{\left\|u_{n}\right\|_{L^{q(x)}\left(\mathbb{R}^{N}\right)}^{q^{+}},\left\|u_{n}\right\|_{L^{q(x)}\left(\mathbb{R}^{N}\right)}^{q^{-}}\right\}\right)^{1 /\left(q / \gamma_{i}\right)^{-}}\right\}\left\|v_{n}\right\|_{L^{q(x)}\left(\mathbb{R}^{N}\right)} \\
+ & 3 \sum_{i=1}^{m}\left\|h_{i}\right\|_{L^{q(x) /\left(q(x)-\gamma_{i}(x)\right)}\left(\mathbb{R}^{N}\right)} \max \left\{\left(\max \left\{\|u\|_{L^{q(x)}\left(\mathbb{R}^{N}\right)}^{q^{+}},\|u\|_{L^{q(x)}\left(\mathbb{R}^{N}\right)}^{q^{-}}\right\}\right)^{1 /\left(q / \gamma_{i}\right)^{+}},\right. \\
& \left.\left(\max \left\{\|u\|_{L^{q(x)}\left(\mathbb{R}^{N}\right)}^{q^{+}},\|u\|_{L^{q(x)}\left(\mathbb{R}^{N}\right)}^{q^{-}}\right\}\right)^{1 /\left(q / \gamma_{i}\right)^{-}}\right\}\left\|v_{n}\right\|_{L^{q(x)}\left(\mathbb{R}^{N}\right)} \\
+ & g_{m+1}\left\|u_{n}\right\|_{L^{2}\left(\mathbb{R}^{N}\right)}\left\|v_{n}\right\|_{L^{2}\left(\mathbb{R}^{N}\right)}+g_{m+1}\|u\|_{L^{2}\left(\mathbb{R}^{N}\right)}\left\|v_{n}\right\|_{L^{2}\left(\mathbb{R}^{N}\right)} \\
+ & \max \left\{\left(\max \left\{\left\|u_{n}\right\|_{L^{q(x)}\left(\mathbb{R}^{N}\right)}^{q^{+}},\left\|u_{n}\right\|_{L^{q(x)}\left(\mathbb{R}^{N}\right)}^{q^{-}}\right\}\right)^{1 /(q /(q-1))^{+}},\right. \\
& \left.\left(\max \left\{\left\|u_{n}\right\|_{L^{q(x)}\left(\mathbb{R}^{N}\right)}^{q^{+}},\left\|u_{n}\right\|_{L^{q(x)}\left(\mathbb{R}^{N}\right)}^{q^{-}}\right\}\right)^{1 /(q /(q-1))^{-}}\right\}\left\|v_{n}\right\|_{L^{q(x)}\left(\mathbb{R}^{N}\right)} \\
+ & \max \left\{\left(\max \left\{\|u\|_{L^{q(x)}\left(\mathbb{R}^{N}\right)}^{q^{+}},\|u\|_{L^{q(x)}\left(\mathbb{R}^{N}\right)}^{q^{-}}\right\}\right)^{1 /(q /(q-1))^{+}},\right. \\
& \left.\quad\left(\max \left\{\|u\|_{L^{q(x)}\left(\mathbb{R}^{N}\right)}^{q^{+}},\|u\|_{L^{q(x)}\left(\mathbb{R}^{N}\right)}^{q^{-}}\right\}\right)^{1 /(q /(q-1))^{-}}\right\}\left\|v_{n}\right\|_{L^{q(x)}\left(\mathbb{R}^{N}\right)} .
\end{aligned}
$$

Since $\left\{u_{n}\right\},\left\{v_{n}\right\}$ are two bounded sequences in $W$, and then they are bounded in $L^{2}\left(\mathbb{R}^{N}\right)$ and $L^{q(x)}\left(\mathbb{R}^{N}\right)$, from that inequality we get $\left|f\left(x, u_{n}\right) v_{n}-f(x, u) v_{n}\right| \in L^{1}\left(\mathbb{R}^{N}\right)$. Hence, there 
exists a constant $A>0$ such that $\left|f\left(x, u_{n}\right) v_{n}-f(x, u) v_{n}\right| \leq A$ for all most everywhere $x \in \mathbb{R}^{N}$. For any $\varepsilon>0$, there exists $\delta=\varepsilon / A$, then for all measurable set $E \subset \mathbb{R}^{N}$ such that $|E|<\delta$, we have

$$
\int_{E}\left|f\left(x, u_{n}\right) v_{n}-f(x, u) v_{n}\right| d x \leq A|E|=\delta A=\varepsilon .
$$

Now, we prove there there exists $R>0$ such that

$$
\int_{\mathbb{R}^{N} \backslash B_{R}}\left|f\left(x, u_{n}\right) v_{n}-f(x, u) v_{n}\right| d x<\varepsilon .
$$

Indeed, since $u_{n} \rightarrow u$ in $L^{q(x)}\left(\mathbb{R}^{N}\right)$, then for any $\varepsilon^{\prime}>0$, we have

$$
\left\|u_{n}-u\right\|_{L^{q(x)}\left(\mathbb{R}^{N}\right)}<\varepsilon^{\prime}
$$

for all $n$ large enough. Note that for $\lambda>0$ such that

$$
\int_{\mathbb{R}^{N} \backslash B_{R}}\left(\frac{\left|u_{n}-u\right|}{\lambda}\right)^{q(x)} d x \leq \int_{\mathbb{R}^{N}}\left(\frac{\left|u_{n}-u\right|}{\lambda}\right)^{q(x)} d x \leq 1,
$$

then by the definition of norm, we have

$$
\begin{aligned}
\left\|u_{n}-u\right\|_{L^{q(x)}\left(\mathbb{R}^{N} \backslash B_{R}\right)} & =\inf \left\{\gamma>0: \int_{\mathbb{R}^{N} \backslash B_{R}}\left(\frac{\left|u_{n}-u\right|}{\gamma}\right)^{q(x)} d x \leq 1\right\} \\
& \leq \inf \left\{\lambda>0: \int_{\mathbb{R}^{N}}\left(\frac{\left|u_{n}-u\right|}{\gamma}\right)^{q(x)} d x \leq 1\right\} \\
& =\left\|u_{n}-u\right\|_{L^{q(x)}\left(\mathbb{R}^{N}\right)} .
\end{aligned}
$$

Combining (2.21) and (2.22), we deduce

$$
\left\|u_{n}\right\|_{L^{q(x)}\left(\mathbb{R}^{N} \backslash B_{R}\right)}-\|u\|_{L^{q(x)}\left(\mathbb{R}^{N} \backslash B_{R}\right)} \leq\left\|u_{n}-u\right\|_{L^{q(x)}\left(\mathbb{R}^{N} \backslash B_{R}\right)}<\varepsilon^{\prime}
$$

for all $n$ large enough. Since $\int_{\mathbb{R}^{N}}\left(\frac{|u|}{\|u\|_{L^{q(x)}\left(\mathbb{R}^{N}\right)}}\right)^{q(x)} d x=1$, then

$$
\int_{\mathbb{R}^{N} \backslash B_{R}}\left(\frac{|u|}{\|u\|_{L^{q(x)}\left(\mathbb{R}^{N}\right)}}\right)^{q(x)} d x \rightarrow 0
$$

as $R \rightarrow+\infty$. Now we will show

$$
\|u\|_{L^{q(x)}\left(\mathbb{R}^{N} \backslash B_{R}\right)} \rightarrow 0
$$

as $R \rightarrow+\infty$. For any small $\eta>0$,

$$
\begin{aligned}
\int_{\mathbb{R}^{N} \backslash B_{R}}\left(\frac{|u|}{\eta}\right)^{q(x)} d x & =\int_{\mathbb{R}^{N} \backslash B_{R}}\left(\frac{\|u\|_{L^{q(x)}\left(\mathbb{R}^{N}\right)}}{\eta}\right)^{q(x)}\left(\frac{|u|}{\|u\|_{L^{q(x)}\left(\mathbb{R}^{N}\right)}}\right)^{q(x)} d x \\
& \leq\left(\frac{\|u\|_{L^{q(x)}\left(\mathbb{R}^{N}\right)}}{\eta}\right)^{q^{+}} \int_{\mathbb{R}^{N} \backslash B_{R}}\left(\frac{|u|}{\|u\|_{L^{q(x)}\left(\mathbb{R}^{N}\right)}}\right)^{q(x)} d x .
\end{aligned}
$$


Combining 2.24 to 2.26, we obtain $\int_{\mathbb{R}^{N} \backslash B_{R}}\left(\frac{|u|}{\eta}\right)^{q(x)} d x \leq 1$ when $R$ is large enough, which implies

$$
\|u\|_{L^{q(x)}\left(\mathbb{R}^{N} \backslash B_{R}\right)}=\inf \left\{\lambda>0: \int_{\mathbb{R}^{N} \backslash B_{R}}\left(\frac{|u|}{\lambda}\right)^{q(x)} d x \leq 1\right\} \leq \eta
$$

for any $\eta>0$. This means that $(2.25)$ is proved and from 2.23$)$, we get

$$
\left\|u_{n}\right\|_{L^{q(x)}\left(\mathbb{R}^{N} \backslash B_{R}\right)} \leq 2 \varepsilon^{\prime}
$$

when $n$ is large enough. By arguments as in (2.27), we also get

$$
\left\|v_{n}\right\|_{L^{q(x)}\left(\mathbb{R}^{N} \backslash B_{R}\right)} \leq 2 \varepsilon^{\prime}
$$

Certainly, we always have

$$
\begin{aligned}
\left\|v_{n}\right\|_{L^{2}\left(\mathbb{R}^{N} \backslash B_{R}\right)} & \leq 2 \varepsilon^{\prime}, \quad\|v\|_{L^{2}\left(\mathbb{R}^{N} \backslash B_{R}\right)} \leq 2 \varepsilon^{\prime} \\
\left\|u_{n}\right\|_{L^{2}\left(\mathbb{R}^{N} \backslash B_{R}\right)} & \leq 2 \varepsilon^{\prime}, \quad\|u\|_{L^{2}\left(\mathbb{R}^{N} \backslash B_{R}\right)} \leq 2 \varepsilon^{\prime}
\end{aligned}
$$

when $n$ is large enough and $R \gg 0$. Similarly, for $R \gg 0$ we get

$$
\left\|h_{0}\right\|_{L^{q(x) /(q(x)-1)}\left(\mathbb{R}^{N} \backslash B_{R}\right)}<2 \varepsilon^{\prime}, \quad\left\|h_{i}\right\|_{L^{q(x) /\left(q(x)-\gamma_{i}(x)\right)}\left(\mathbb{R}^{N} \backslash B_{R}\right)}<2 \varepsilon^{\prime}
$$

for all $i=1, \ldots, m$ and $R \gg 0$. Choosing a common $R \gg 0$ from 2.25, 2.27)-2.30 and $\varepsilon^{\prime}>0$ small enough, we get 2.20$)$. By 2.19$)$ and 2.20$)$, we get $\int_{\mathbb{R}^{N}} \mid f\left(x, u_{n}\right) v_{n}-$ $f(x, u) v_{n} \mid d x$ is equi-integrable. Applying Vitali's theorem, we get

$$
\lim _{n \rightarrow \infty} \int_{\mathbb{R}^{N}}\left|f\left(x, u_{n}\right) v_{n}-f(x, u) v_{n}\right| d x=0,
$$

which is a contradiction. Then $\rho=0$, so

$$
\left\|\psi^{\prime}\left(u_{n}\right)-\psi^{\prime}(u)\right\|=\sup _{\|v\|_{W}=1}\left|\int_{\mathbb{R}^{N}}\left(f\left(x, u_{n}\right) v-f(x, u) v\right) d x\right| \rightarrow 0
$$

as $n \rightarrow \infty$. Thus, $\psi^{\prime}$ is weakly continuous. The compactness of $\psi^{\prime}$ follows from its weak continuity and $W$ is a reflexive space.

In order to study problem (1.1), we use the energy as follows:

$$
I(u)=\frac{1}{2}\left([u]_{s(\cdot)}^{2}+\int_{\mathbb{R}^{N}} V(x)|u|^{2} d x\right)-\lambda \int_{\mathbb{R}^{N}} F(x, u) d x
$$

then $I \in C^{1}(W, \mathbb{R})$ by Lemma 2.11 and

$$
\left\langle I^{\prime}(u), v\right\rangle=\int_{\mathbb{R}^{2 N}} \frac{(u(x)-u(y))(v(x)-v(y))}{|x-y|^{N+2 s(x, y)}} d x d y+\int_{\mathbb{R}^{N}} V(x) u v d x-\lambda \int_{\mathbb{R}^{N}} f(x, u) v d x
$$

for all $u, v \in W$. 


\section{Proof of Theorem 1.4}

The compactness assumption required by the Fountain Theorem is the well-known PalaisSmale condition (see, for instance, 21,22 and references therein), which in our framework reads as follows:

Palais-Smale condition. The functional I satisfies the Palais-Smale compactness condition at level $c \in \mathbb{R}$ if any sequence $\left\{u_{j}\right\}_{j \in \mathbb{N}}$ in $W$ such that $I\left(u_{j}\right) \rightarrow c$ and $\sup _{\|\varphi\|_{W}=1}$ $\left|\left\langle I^{\prime}\left(u_{j}\right), \varphi\right\rangle\right| \rightarrow 0$, admits a strongly convergent subsequence in $W$.

In order to prove the theorems in this paper, we need the Mountain Pass Theorem as follows:

Lemma 3.1. 19 Let $X$ be a real Banach space and $I \in C^{1}(X, \mathbb{R})$ satisfy Palais-Smale condition. Suppose that $I(0)=0$ and

$\left(\mathrm{I}_{1}\right)$ there exist two constants $\beta_{0}, \alpha_{0}>0$ such that $I(u) \geq \alpha_{0}$ for all $\|u\|=\beta_{0}$,

$\left(\mathrm{I}_{2}\right)$ there is $u_{1} \in X,\left\|u_{1}\right\| \geq \beta_{0}$ such that $I\left(u_{1}\right) \leq 0$.

Then I possesses a critical value $c \geq \alpha_{0}$. Moreover, $c$ can be characterized as

$$
c=\inf _{\gamma \in \Gamma} \max _{t \in[0,1]} I(\gamma(t)),
$$

where $\Gamma=\left\{\gamma \in C([0,1], X): \gamma(0)=0, \gamma(1)=u_{1}\right\}$.

Lemma 3.2. Let $\left(\mathrm{f}_{1}\right)$ and $\left(\mathrm{f}_{2}\right),\left(\mathrm{V}_{1}\right)$ hold. Then there exist positive constants $t_{0}, \alpha_{0}$ such that $I(u) \geq \alpha_{0}$ for all $u \in W$ with $\|u\|_{W}=t_{0}$ for any fixed $\lambda>0$.

Proof. By Remark 1.1, for any $\varepsilon>0$, there exists $C_{\varepsilon}>0$ such that

$$
|f(x, t)| \leq \varepsilon|t|+C_{\varepsilon}|t|^{q(x)-1}
$$

for any $x \in \mathbb{R}^{N}, t \in \mathbb{R}$. Then from 3.1 , we get

$$
F(x, t) \leq \varepsilon|t|^{2}+C_{\varepsilon} \frac{|t|^{q(x)}}{q(x)}
$$

for any $x \in \mathbb{R}^{N}, t \in \mathbb{R}$, and for some $C_{\varepsilon}>0$. For $u \in W,\|u\|_{W}=\rho \in(0,1)$, we have

$$
\begin{aligned}
I(u) & =\frac{1}{2}\left([u]_{s(\cdot)}^{2}+\|u\|_{2, V}^{2}\right)-\lambda \int_{\mathbb{R}^{N}} F(x, u) d x \\
& \geq \frac{1}{2}\left([u]_{s(\cdot)}^{2}+\|u\|_{2, V}^{2}\right)-\lambda \varepsilon \int_{\mathbb{R}^{N}}|u|^{2} d x-\lambda C_{\varepsilon} \int_{\mathbb{R}^{N}} \frac{|u|^{q(x)}}{q(x)} d x \\
& \geq \frac{1}{2}\left([u]_{s(\cdot)}^{2}+\|u\|_{2, V}^{2}\right)-\lambda \varepsilon \int_{\mathbb{R}^{N}}|u|^{2} d x-\frac{\lambda C_{\varepsilon}}{q^{-}} \int_{\mathbb{R}^{N}}|u|^{q(x)} d x .
\end{aligned}
$$


From Corollary 2.10, we choose $\rho$ small enough such that

$$
\|u\|_{L^{q(x)}\left(\mathbb{R}^{N}\right)} \leq S_{q}\|u\|_{W}=\rho S_{q} \in(0,1)
$$

and we obtain

$$
\int_{\mathbb{R}^{N}}|u|^{q(x)} d x \leq\|u\|_{L^{q(x)}\left(\mathbb{R}^{N}\right)}^{q^{-}} \leq S_{q}^{q^{-}}\|u\|_{W}^{q^{-}} .
$$

Using Corollary 2.10 again, (3.2) and (3.3), we get

$$
\begin{aligned}
I(u) & \geq \frac{1}{2}\|u\|_{W}^{2}-\varepsilon \lambda S_{2}^{2}\|u\|_{W}^{2}-\frac{\lambda C_{\varepsilon} S_{q}^{q^{-}}}{q^{-}}\|u\|_{W}^{q^{-}} \\
& =\|u\|_{W}^{2}\left(\left(\frac{1}{2}-\varepsilon \lambda S_{2}^{2}\right)-\frac{\lambda C_{\varepsilon} S_{q}^{q^{-}}}{q^{-}}\|u\|_{W}^{q^{-}-2}\right) .
\end{aligned}
$$

We choose $\varepsilon>0$ enough small such that $\frac{1}{2}-\varepsilon \lambda S_{2}^{2}>0$ for any fixed $\lambda>0$. Let $h(t)=$ $\frac{1}{2}-\varepsilon \lambda S_{2}^{2}-\frac{\lambda C_{\varepsilon} S_{q}^{q^{-}}}{q^{-}} t^{q^{-}-2}, t \geq 0$. We now prove there exists $t_{0}>0$ small satisfying $h\left(t_{0}\right) \geq$ $\frac{1}{2}\left(\frac{1}{2}-\varepsilon \lambda S_{2}^{2}\right)$. We see that $h$ is continuous function on $[0,+\infty)$ and $\lim _{t \rightarrow 0^{+}} h(t)=\frac{1}{2}-\varepsilon \lambda S_{2}^{2}$, then there exists $0<t_{0}<\rho$ such that $h(t) \geq \frac{1}{2}-\varepsilon \lambda S_{2}^{2}-\varepsilon_{1}$ for all $0 \leq t \leq t_{0}$. If we choose $\varepsilon_{1}=\frac{1}{2}\left(\frac{1}{2}-\varepsilon \lambda S_{2}^{2}\right)$, we have

$$
h(t) \geq \frac{1}{2}\left(\frac{1}{2}-\varepsilon \lambda S_{2}^{2}\right)
$$

for all $0 \leq t \leq t_{0}$. Especially,

$$
h\left(t_{0}\right) \geq \frac{1}{2}\left(\frac{1}{2}-\varepsilon \lambda S_{2}^{2}\right)
$$

From (3.4) and (3.5), for $\|u\|_{W}=t_{0}$, we have

$$
I(u) \geq \frac{t_{0}^{2}}{2}\left(\frac{1}{2}-\varepsilon \lambda S_{2}^{2}\right)=\alpha_{0}
$$

for $\|u\|_{W}=t_{0}$.

Lemma 3.3. Suppose that $\left(\mathrm{V}_{1}\right)$ and $\left(\mathrm{f}_{4}\right)$ hold. Then there exists a function $v \in W$ with $\|v\|_{W}>t_{0}$, such that $I(v)<0$, where $t_{0}>0$ is the number given in Lemma 3.2 .

Proof. For all $u \in W$ with $\|u\|_{W}=1$ and all $t>1$, from the condition $\left(\mathrm{f}_{4}\right)$, we obtain

$$
\begin{aligned}
I(t u) & =\frac{1}{2}\left([t u]_{s(\cdot)}^{2}+\|t u\|_{2, V}^{2}\right)-\lambda \int_{\mathbb{R}^{N}} F(x, t u) d x \\
& \leq \frac{t^{2}}{2}-\lambda C_{\mu} t^{\mu} \int_{\mathbb{R}^{N}}|u(x)|^{\mu} d x+\lambda \int_{\mathbb{R}^{N}} P(t|u|) d x,
\end{aligned}
$$


where $P$ is a function on $\mathbb{R}$ such that

$$
P(t)=a_{n}|t|^{\alpha_{n}(x)}+\cdots+a_{1}|t|^{\alpha_{1}(x)}+a_{0} \quad \text { for all } t \in \mathbb{R}
$$

where $n \in \mathbb{N}, a_{i} \in \mathbb{R}, i=0, \ldots, n, a_{n} \neq 0$ and $+\infty>\alpha_{n}(x)>\cdots>\alpha_{1}(x)>0, \mu>\alpha_{n}^{+} \geq$ $\alpha_{n}(x) \geq 2$ for all $x \in \mathbb{R}^{N}$ and $a_{0} \leq 0, a_{i}<0$ if $\alpha_{i} \in(0,2)$ for some $i \in\{1, \ldots, n-1\}$ if $n \geq 2$. We can write $P=P_{1}+Q_{1}$, where $P_{1}$ is a function with the exponent function $\alpha(x)$ such that $2 \leq \alpha(x)<\mu$ for all $x \in \mathbb{R}^{N}$ and $Q_{1}$ is a function with exponent function $\alpha(x)$ such that $0 \leq \alpha(x)<2$ for all $x \in \mathbb{R}^{N}$, where $\alpha \in\left\{0, \alpha_{1}, \ldots, \alpha_{n}\right\}$. Since all coefficients of $Q_{1}$ are negative, then $\int_{\mathbb{R}^{N}} Q_{1}(|t u|) d x<0$. For all $\alpha, 2 \leq \alpha(x) \leq \alpha_{n}(x)<\mu$ for all $x \in \mathbb{R}^{N}$, we have $\inf _{x \in \mathbb{R}^{N}}\left(2_{s}^{*}(x)-\alpha(x)\right)>0$ and $\alpha \in C_{+}\left(\mathbb{R}^{N}\right)$ is a uniformly continuous function, applying Lemma 2.3 , then we get

$$
\|u\|_{L^{\alpha(x)}\left(\mathbb{R}^{N}\right)} \leq S_{\alpha}\|u\|_{W}=S_{\alpha}<+\infty
$$

Note that

$$
\begin{aligned}
\int_{\mathbb{R}^{N}}|t u|^{\alpha(x)} d x & \leq t^{\alpha^{+}} \int_{\mathbb{R}^{N}}|u|^{\alpha(x)} d x \\
& \leq t^{\alpha^{+}} \max \left\{\|u\|_{L^{\alpha(x)}\left(\mathbb{R}^{N}\right)}^{\alpha^{+}},\|u\|_{L^{\alpha(x)}\left(\mathbb{R}^{N}\right)}^{\alpha^{-}}\right\} \leq t^{\alpha^{+}} \max \left\{S_{\alpha}^{\alpha^{+}}, S_{\alpha}^{\alpha^{-}}\right\}
\end{aligned}
$$

then $\int_{\mathbb{R}^{N}}|t u|^{\alpha(x)} d x$ has growth smaller than $t^{\mu}$ as $t \rightarrow \infty$. Furthermore, we also have $\int_{\mathbb{R}^{N}}|u|^{\mu} d x \leq S_{\mu}^{\mu}\|u\|_{W}^{\mu}=S_{\mu}^{\mu}<+\infty$. Since $\mu>2, \mu>\alpha_{n}^{+}$, we have $I(t u) \rightarrow-\infty$ as $t \rightarrow+\infty$. Taking $v=\rho_{0} u, \rho_{0}>t_{0}>0$ large enough, we have $I(v)<0,\|v\|_{W}>t_{0}$.

Lemma 3.4. Suppose that $V$ satisfies the conditions $\left(\mathrm{V}_{1}\right)$ and $\left(\mathrm{V}_{2}\right)$ or $\left(\mathrm{V}_{1}\right)$ and $\left(\mathrm{V}_{3}\right)$, $f$ satisfies the conditions $\left(\mathrm{f}_{1}\right),\left(\mathrm{f}_{2}\right)$ and $\left(\mathrm{f}_{3}\right)$. Then I satisfies the Palais-Smale condition at level $c \in \mathbb{R}$ for all $\lambda$ satisfying $[1.2$.

Proof. Let $\left\{u_{n}\right\}$ be a (PS) sequence with level $c \in \mathbb{R}$ in $W$. This means

$$
I\left(u_{n}\right) \rightarrow c \quad \text { and } \quad \sup _{\|\varphi\|_{W}=1}\left|\left\langle I^{\prime}\left(u_{n}\right), \varphi\right\rangle\right| \rightarrow 0
$$

as $n \rightarrow \infty$. We split the proof into two steps. First, we show that the sequence $\left\{u_{n}\right\}$ is bounded in $W$ and that it admits a subsequence strongly convergent in $W$. From (3.6), for any $n \in \mathbb{N}$, there exists $C>0$ such that

$$
\left|\left\langle I^{\prime}\left(u_{n}\right), \frac{u_{n}}{\left\|u_{n}\right\|_{W}}\right\rangle\right| \leq K \quad \text { and } \quad\left|I\left(u_{n}\right)\right| \leq C
$$

so that

$$
I\left(u_{n}\right)-\mu\left\langle I^{\prime}\left(u_{n}\right), u_{n}\right\rangle \leq C\left(1+\left\|u_{n}\right\|_{W}\right),
$$


where $\mu$ is a parameter in the condition $\left(\mathrm{f}_{3}\right)$. Set $A_{n}=\left\{x \in \mathbb{R}^{N}:\left|u_{n}(x)\right| \leq r\right\}$. We have

$$
I\left(u_{n}\right)-\frac{1}{\mu}\left\langle I^{\prime}\left(u_{n}\right), u_{n}\right\rangle=\left(\frac{1}{2}-\frac{1}{\mu}\right)\left\|u_{n}\right\|_{W}^{2}-\frac{\lambda}{\mu} \int_{\mathbb{R}^{N}}\left(f\left(x, u_{n}\right)-\mu F\left(x, u_{n}\right)\right) d x .
$$

Therefore, we deduce

$$
\begin{aligned}
I\left(u_{n}\right)-\frac{1}{\mu}\left\langle I^{\prime}\left(u_{n}\right), u_{n}\right\rangle= & \left(\frac{1}{2}-\frac{1}{\mu}\right)\left\|u_{n}\right\|_{W}^{2}+\frac{\lambda}{\mu} \int_{A_{n}}\left(f\left(x, u_{n}\right) u_{n}-\mu F\left(x, u_{n}\right)\right) d x \\
& +\frac{\lambda}{\mu} \int_{\mathbb{R}^{N} \backslash A_{n}}\left(f\left(x, u_{n}\right) u_{n}-\mu F\left(x, u_{n}\right)\right) d x \\
\geq & \left(\frac{1}{2}-\frac{1}{\mu}\right)\left\|u_{n}\right\|_{W}^{2}+\frac{\lambda}{\mu} \int_{A_{n}}\left(f\left(x, u_{n}\right) u_{n}-\mu F\left(x, u_{n}\right)\right) d x \\
& -\frac{\lambda \rho}{\mu V^{-}} \int_{\mathbb{R}^{N}} V(x)\left|u_{n}(x)\right|^{2} d x+O(1) .
\end{aligned}
$$

From the assumption $(1.2)$, there exists $n_{0}$ such that

$$
\frac{\lambda \rho}{\mu V^{-}}<\left(1-\frac{1}{n_{0}}\right)\left(\frac{1}{2}-\frac{1}{\mu}\right)
$$

We denote $\kappa=\left(1-\frac{1}{n_{0}}\right)\left(\frac{1}{2}-\frac{1}{\mu}\right)-\frac{\lambda \rho}{\mu V^{-}}$. From $\left(\mathrm{f}_{1}\right)$ and $\left(\mathrm{f}_{2}\right)$, we obtain

$$
|f(x, t)| \leq 2 \varepsilon|t|+C_{\varepsilon}|t|^{q(x)-1}
$$

for all $(x, t) \in \mathbb{R}^{N} \times \mathbb{R}$. Then, on $A_{n}$, we have $\frac{\lambda}{\mu}\left|f\left(x, u_{n}\right) u_{n}-\mu F\left(x, u_{n}\right)\right| \leq \frac{\lambda}{\mu}\left(\varepsilon(2+\mu)+C_{\varepsilon}(1+\mu) \max \left\{r^{q^{+}-2}, r^{q^{-}-2}\right\}\right)\left|u_{n}\right|^{2}=C_{1}\left|u_{n}\right|^{2}$. Take $M>\max \left\{\frac{C_{1}}{\kappa}, V^{-}\right\}$, and let $B_{1}=\left\{x \in B_{h}(y): V(x) \leq M\right\}$ if $V$ satisfies $\left(\mathrm{V}_{2}\right)$ and $B_{2}=\left\{x \in \mathbb{R}^{N}: V(x) \leq M\right\}$ if $V$ satisfies $\left(\mathrm{V}_{3}\right)$. By the assumptions $\left(\mathrm{V}_{2}\right)$ and $\left(\mathrm{V}_{3}\right)$, we have meas $\left(B_{1}\right)<+\infty$ for all $y \in \mathbb{R}^{N}$ and meas $\left(B_{2}\right)<+\infty$, respectively. Then we obtain

$$
\begin{aligned}
& \kappa \int_{\mathbb{R}^{N}} V(x)\left|u_{n}\right|^{2} d x+\frac{\lambda}{\mu} \int_{A_{n}}\left(f\left(x, u_{n}\right) u_{n}-\mu F\left(x, u_{n}\right)\right) d x \\
\geq & \kappa \int_{\mathbb{R}^{N}} V(x)\left|u_{n}\right|^{2} d x-C_{1} \int_{A_{n}}\left|u_{n}\right|^{2} d x \\
\geq & \kappa \int_{\mathbb{R}^{N}} V(x)\left|u_{n}\right|^{2} d x-M \kappa \int_{A_{n}}\left|u_{n}\right|^{2} d x \\
= & \kappa \int_{A_{n}}(V(x)-M)\left|u_{n}\right|^{2} d x \geq \kappa \int_{A_{n} \cap B_{i}}(V(x)-M) r^{2} d x \\
\geq & \kappa\left(V^{-}-M\right) r^{2} \operatorname{meas}\left(A_{n} \cap B_{i}\right) \geq \kappa\left(V^{-}-M\right) r^{2} \operatorname{meas}\left(B_{i}\right),
\end{aligned}
$$

where $i=1$ or $i=2$. On combining 3.7$)$ to 3.9 , we have

$$
C\left(1+\left\|u_{n}\right\|_{W}\right) \geq \frac{1}{n_{0}}\left(\frac{1}{2}-\frac{1}{\mu}\right)\left\|u_{n}\right\|_{W}^{2}+\kappa\left(V^{-}-M\right) r^{2} \operatorname{meas}\left(B_{i}\right) .
$$


From (3.10), we conclude that the sequence $\left\{u_{n}\right\}$ is bounded in $W$. Going if necessary to a subsequence, thanks to Lemmas 2.6 and 2.9 , we have

$$
u_{n} \rightarrow u \text { weakly in } W, \quad u_{n} \rightarrow u \text { in } L^{2}\left(\mathbb{R}^{N}\right) \text { and } L^{q(x)}\left(\mathbb{R}^{N}\right), \quad u_{n} \rightarrow u \text { in } \mathbb{R}^{N} .
$$

We denote $B_{\phi}$ by the linear function on $H_{\lambda}$ as follows:

$$
B_{\phi}(\varphi)=\int_{\mathbb{R}^{2 N}} \frac{(\phi(x)-\phi(y))(\varphi(x)-\varphi(y))}{|x-y|^{N+2 s(x, y)}} d x+\int_{\mathbb{R}^{N}} V(x) \phi \varphi d x .
$$

Clearly, by the Hölder inequality, $B_{\phi}$ is a continuous linearly mapping on $W$ and

$$
\left|B_{\phi}(\varphi)\right| \leq 2\|\phi\|_{W}\|\varphi\|_{W} \quad \text { for all } \varphi \in W
$$

From (3.8) and using the Hölder inequality, we get

$$
\begin{aligned}
& \int_{\mathbb{R}^{N}}\left|\left(f\left(x, u_{n}\right)-f(x, u)\right)\left(u_{n}-u\right)\right| d x \\
\leq & \int_{\mathbb{R}^{N}}\left[2 \varepsilon\left|u_{n}\right|+2 \varepsilon|u|+C_{\varepsilon}\left(\left|u_{n}\right|^{q(x)-1}+|u|^{q(x)-1}\right)\right]\left|u_{n}-u\right| d x \\
\leq & 2 \varepsilon\left(\left\|u_{n}\right\|_{L^{2}\left(\mathbb{R}^{N}\right)}+\|u\|_{L^{2}\left(\mathbb{R}^{N}\right)}\right)\left\|u_{n}-u\right\|_{L^{2}\left(\mathbb{R}^{N}\right)} \\
& +2 C_{\varepsilon}\left(\left\|\left|u_{n}\right|^{q(x)}\right\|_{L^{q(x) /(q(x)-1)}\left(\mathbb{R}^{N}\right)}+\left\||u|^{q(x)}\right\|_{L^{q(x) /(q(x)-1)}\left(\mathbb{R}^{N}\right)}\right)\left\|u_{n}-u\right\|_{L^{q(x)}\left(\mathbb{R}^{N}\right)}
\end{aligned}
$$

Since $u_{n} \rightarrow u$ in $L^{q(x)}\left(\mathbb{R}^{N}\right)$, then by Brezis-Lieb Lemma, $\left|u_{n}\right|^{q(x)-1} \rightarrow|u|^{q(x)-1}$ in $L^{q(x) /(q(x)-1)}\left(\mathbb{R}^{N}\right)$. This implies $\left\|\left|u_{n}\right|^{q(x)}\right\|_{L^{q(x) /(q(x)-1)}\left(\mathbb{R}^{N}\right)}$ is a bounded sequence. Hence from 3.11 , we get

$$
\lim _{n \rightarrow \infty} \int_{\mathbb{R}^{N}}\left(f\left(x, u_{n}\right)-f(x, u)\right)\left(u_{n}-u\right) d x=0 .
$$

Obviously, $\left\langle I^{\prime}\left(u_{n}\right)-I^{\prime}(u), u_{n}-u\right\rangle \rightarrow 0$ since $u_{n} \rightarrow u$ weakly in $W$ and $I^{\prime}\left(u_{n}\right) \rightarrow 0$. Therefore, from 3.12 , we obtain

$$
\begin{aligned}
o(1) & =\left\langle I^{\prime}\left(u_{n}\right)-I^{\prime}(u), u_{n}-u\right\rangle \\
& =B_{u_{n}}\left(u_{n}-u\right)-B_{u}\left(u_{n}-u\right)-\lambda \int_{\mathbb{R}^{N}}\left(f\left(x, u_{n}\right)-f(x, u)\right)\left(u_{n}-u\right) d x \\
& =B_{u_{n}}\left(u_{n}-u\right)-B_{u}\left(u_{n}-u\right)+o(1)
\end{aligned}
$$

for all $n$ large enough. We note that $B_{u_{n}}\left(u_{n}-u\right)-B_{u}\left(u_{n}-u\right)=\left\|u_{n}-u\right\|_{W}^{2}$, then we deduce $u_{n} \rightarrow u$ in $W$ from (3.13). Combining Lemma 2.8 to Lemma 3.4, we get the existence of a nontrivial week solution to problem (1.1). 


\section{Proof of Theorem 1.5}

In order to prove Theorem 1.5 , we need the following lemma.

Lemma 4.1. Suppose that $V$ satisfies the conditions $\left(\mathrm{V}_{1}\right)$ and $\left(\mathrm{V}_{2}\right)$ or $\left(\mathrm{V}_{1}\right)$ and $\left(\mathrm{V}_{3}\right)$, $f$ satisfies the conditions $(\mathfrak{f})$ and $\left(\mathrm{f}_{3}\right)$. Then I satisfies the Palais-Smale condition at level $c \in \mathbb{R}$ for all $\lambda$ satisfying 1.2 .

Proof. Let $\left\{u_{n}\right\}$ be a (PS) sequence with level $c \in \mathbb{R}$ in $W$. This means

$$
I\left(u_{n}\right) \rightarrow c \quad \text { and } \quad \sup _{\|\varphi\|_{W}=1}\left|\left\langle I^{\prime}\left(u_{n}\right), \varphi\right\rangle\right| \rightarrow 0
$$

as $n \rightarrow \infty$. We split the proof into two steps. First, we show that the sequence $\left\{u_{n}\right\}$ is bounded in $W$ and that it admits a subsequence strongly convergent in $W$. From (4.1), for any $n \in \mathbb{N}$, there exists $C>0$ such that

$$
\left|\left\langle I^{\prime}\left(u_{n}\right), \frac{u_{n}}{\left\|u_{n}\right\|_{W}}\right\rangle\right| \leq K \quad \text { and } \quad\left|I\left(u_{n}\right)\right| \leq C
$$

so that

$$
I\left(u_{n}\right)-\frac{1}{\mu}\left\langle I^{\prime}\left(u_{n}\right), u_{n}\right\rangle \leq C\left(1+\left\|u_{n}\right\|_{W}\right)
$$

where $\mu$ is a parameter in the condition $\left(\mathrm{f}_{3}\right)$. Set $A_{n}=\left\{x \in \mathbb{R}^{N}:\left|u_{n}(x)\right| \leq r\right\}$. We have

$$
\begin{aligned}
I\left(u_{n}\right)-\frac{1}{\mu}\left\langle I^{\prime}\left(u_{n}\right), u_{n}\right\rangle= & \frac{1}{2}\left\|u_{n}\right\|_{W}^{2}-\lambda \int_{\mathbb{R}^{N}} F\left(x, u_{n}\right) d x \\
& -\frac{1}{\mu}\left[\left\|u_{n}\right\|_{W}^{2}-\lambda \int_{\mathbb{R}^{N}} f\left(x, u_{n}\right) u_{n} d x\right] .
\end{aligned}
$$

Hence, we have

$$
\begin{aligned}
I\left(u_{n}\right)-\frac{1}{\mu}\left\langle I^{\prime}\left(u_{n}\right), u_{n}\right\rangle= & \left(\frac{1}{2}-\frac{1}{\mu}\right)\left\|u_{n}\right\|_{W}^{2}+\frac{\lambda}{\mu} \int_{A_{n}}\left(f\left(x, u_{n}\right) u_{n}-\mu F\left(x, u_{n}\right)\right) d x \\
& +\frac{\lambda}{\mu} \int_{\mathbb{R}^{N} \backslash A_{n}}\left(f\left(x, u_{n}\right) u_{n}-\mu F\left(x, u_{n}\right)\right) d x \\
\geq & \left(\frac{1}{2}-\frac{1}{\mu}\right)\left\|u_{n}\right\|_{W}^{2}+\frac{\lambda}{\mu} \int_{A_{n}}\left(f\left(x, u_{n}\right) u_{n}-\mu F\left(x, u_{n}\right)\right) d x \\
& -\frac{\lambda \rho}{\mu} \int_{\mathbb{R}^{N}}\left|u_{n}(x)\right|^{2} d x-\frac{\lambda}{\mu} \int_{\mathbb{R}^{N}} \varphi_{*}(x) d x .
\end{aligned}
$$

Therefore, we deduce

$$
\begin{aligned}
I\left(u_{n}\right)-\frac{1}{\mu}\left\langle I^{\prime}\left(u_{n}\right), u_{n}\right\rangle \geq & \left(\frac{1}{2}-\frac{1}{\mu}\right)\left\|u_{n}\right\|_{W}^{2}+\frac{\lambda}{\mu} \int_{A_{n}}\left(f\left(x, u_{n}\right) u_{n}-\mu F\left(x, u_{n}\right)\right) d x \\
& -\frac{\lambda \rho}{\mu V^{-}} \int_{\mathbb{R}^{N}} V(x)\left|u_{n}(x)\right|^{2} d x+O(1) .
\end{aligned}
$$


From the assumption 1.2 , there exists $n_{0}$ such that

$$
\frac{\lambda \rho}{\mu V^{-}}<\left(1-\frac{1}{n_{0}}\right)\left(\frac{1}{2}-\frac{1}{\nu}\right) .
$$

Denote $\kappa=\left(1-\frac{1}{n_{0}}\right)\left(\frac{1}{2}-\frac{1}{\mu}\right)-\frac{\lambda \rho}{\mu V^{-}}$. From the condition $(\mathfrak{f})$, we have

$$
\begin{aligned}
& \left|f\left(x, u_{n}\right) u_{n}-\mu F\left(x, u_{n}\right)\right| \\
\leq & (\mu+1)\left(\left|h_{0}(x)\right|\left|u_{n}\right|+\sum_{i=1}^{m}\left|h_{i}(x)\right|\left|u_{n}\right|^{\gamma_{i}(x)}+g_{m+1}\left|u_{n}\right|^{2}+h_{m+1}\left|u_{n}\right|^{q(x)}\right) .
\end{aligned}
$$

Then, on $A_{n}$, using Hölder inequality and Corollary 2.10, we see

$$
\begin{aligned}
& \quad \frac{\lambda}{\mu} \int_{A_{n}}\left|f\left(x, u_{n}\right) u_{n}-\mu F\left(x, u_{n}\right)\right| d x \\
& \leq \frac{\lambda}{\mu}(\mu+1)\left(2\left\|h_{0}\right\|_{L^{q(x) /(q(x)-1)}}\left\|u_{n}\right\|_{L^{q(x)}\left(\mathbb{R}^{N}\right)}+g_{m+1}\left\|u_{n}\right\|_{L^{2}\left(\mathbb{R}^{N}\right)}^{2}\right. \\
& \quad+2 \sum_{i=1}^{m}\left\|h_{i}\right\|_{L^{q(x) /\left(q(x)-\gamma_{i}(x)\right)}\left(\mathbb{R}^{N}\right)} \\
& \left.\quad \times \max \left\{\left(\int_{A_{n}}\left|u_{n}\right|^{q(x)} d x\right)^{1 /\left(q / \gamma_{i}\right)^{+}},\left(\int_{A_{n}}\left|u_{n}\right|^{q(x)} d x\right)^{1 /\left(q / \gamma_{i}\right)^{-}}\right\}\right) \\
& +\frac{\lambda}{\mu}(\mu+1)\left(g_{m+1} \int_{A_{n}}\left|u_{n}(x)\right|^{2} d x+h_{m+1} \int_{A_{n}}\left|u_{n}(x)\right|^{q(x)} d x\right) .
\end{aligned}
$$

Thus,

$$
\begin{aligned}
& \frac{\lambda}{\mu} \int_{A_{n}}\left|f\left(x, u_{n}\right) u_{n}-\mu F\left(x, u_{n}\right)\right| d x \\
\leq & 2 \frac{\lambda}{\mu}(\mu+1) S_{q}\left\|h_{0}\right\|_{L^{q(x) /(q(x)-1)\left(\mathbb{R}^{N}\right)}\|u\|_{W}} \\
& +2 \frac{\lambda}{\mu}(\mu+1) \sum_{i=1}^{m}\left\|h_{i}\right\|_{L^{q(x) /\left(q(x)-\gamma_{i}(x)\right)}\left(\mathbb{R}^{N}\right)} \\
& \times\left(\left(\int_{A_{n}} r^{\max \left\{q^{+}, q^{-}\right\}-2}\left|u_{n}\right|^{2} d x\right)^{1 /\left(q / \gamma_{i}\right)^{+}}+\left(\int_{A_{n}} r^{\max \left\{q^{+}, q^{-}\right\}-2}\left|u_{n}\right|^{2} d x\right)^{1 /\left(q / \gamma_{i}\right)^{-}}\right) \\
& +\frac{\lambda}{\mu}(\mu+1)\left(h_{m+1} r^{\max \left\{q^{+}, q^{-}\right\}-2}+g_{m+1}\right) \int_{A_{n}}\left|u_{n}(x)\right|^{2} d x .
\end{aligned}
$$

Therefore, there exists a constant $\mathcal{C}(r)>0$ such that

$$
\begin{aligned}
& \frac{\lambda}{\mu} \int_{A_{n}}\left|f\left(x, u_{n}\right) u_{n}-\mu F\left(x, u_{n}\right)\right| d x \\
\leq & 2 \frac{\lambda}{\mu}(\mu+1) S_{q}\left\|h_{0}\right\|_{L^{q(x) /(q(x)-1)}\left(\mathbb{R}^{N}\right)}\left\|u_{n}\right\|_{W}
\end{aligned}
$$




$$
\begin{aligned}
& +2 \frac{\lambda}{\mu}(\mu+1) \sum_{i=1}^{m}\left\|h_{i}\right\|_{L^{q(x) /\left(q(x)-\gamma_{i}(x)\right)\left(\mathbb{R}^{N}\right)}} \mathcal{C}(r)\left(\left\|u_{n}\right\|_{W}^{2 /\left(\left(q / \gamma_{i}\right)^{+}\right)}+\left\|u_{n}\right\|_{W}^{2 /\left(\left(q / \gamma_{i}\right)^{-}\right)}\right) \\
& +\frac{\lambda}{\mu}(\mu+1)\left(h_{m+1} r^{\max \left\{q^{+}, q^{-}\right\}-2}+g_{m+1}\right) \int_{A_{n}}\left|u_{n}(x)\right|^{2} d x .
\end{aligned}
$$

Set $C_{1}=\frac{\lambda}{\mu}(\mu+1)\left(h_{m+1} r^{\max \left\{q^{+}, q^{-}\right\}-2}+g_{m+1}\right)$, and take $M>\max \left\{\frac{C_{1}}{\kappa}, V^{-}\right\}$. Let $B_{1}=\left\{x \in B_{h}(y): V(x) \leq M\right\}$ if $V$ satisfies $\left(\mathrm{V}_{2}\right)$ and $B_{2}=\left\{x \in \mathbb{R}^{N}: V(x) \leq M\right\}$ if $V$ satisfies the condition $\left(\mathrm{V}_{3}\right)$. By the assumptions $\left(\mathrm{V}_{2}\right)$ and $\left(\mathrm{V}_{3}\right)$, we have meas $\left(B_{1}\right)<+\infty$ for all $y \in \mathbb{R}^{N}$ and meas $\left(B_{2}\right)<+\infty$, respectively. By arguments as in Lemma 3.4, we have

$$
\kappa \int_{\mathbb{R}^{N}} V(x)\left|u_{n}\right|^{2} d x-C_{1} \int_{A_{n}}\left|u_{n}\right|^{2} d x \geq \kappa\left(V^{-}-M\right) r^{2} \operatorname{meas}\left(B_{i}\right)
$$

for $i=1,2$. Combining (4.3) and 4.4 , we get

$$
\begin{aligned}
& \kappa \int_{\mathbb{R}^{N}} V(x)\left|u_{n}\right|^{2} d x+\frac{\lambda}{\mu} \int_{A_{n}}\left(f\left(x, u_{n}\right) u_{n}-\mu F\left(x, u_{n}\right)\right) d x \\
\geq & \kappa \int_{\mathbb{R}^{N}} V(x)\left|u_{n}\right|^{2} d x-C_{1} \int_{A_{n}}\left|u_{n}\right|^{2} d x-\frac{2 \lambda}{\mu}(\mu+1) S_{q}\left\|h_{0}\right\|_{L^{q(x) /(q(x)-1)}\left(\mathbb{R}^{N}\right)}\left\|u_{n}\right\|_{W} \\
& -2 \frac{\lambda}{\mu}(\mu+1) \sum_{i=1}^{m}\left\|h_{i}\right\|_{L^{q(x) /\left(q(x)-\gamma_{i}(x)\right)\left(\mathbb{R}^{N}\right)}} \mathcal{C}(r)\left(\left\|u_{n}\right\|_{W}^{2 /\left(\left(q / \gamma_{i}\right)^{+}\right)}+\left\|u_{n}\right\|_{W}^{2 /\left(\left(q / \gamma_{i}\right)^{-}\right)}\right) .
\end{aligned}
$$

That inequality implies

$$
\begin{aligned}
& \kappa \int_{\mathbb{R}^{N}} V(x)\left|u_{n}\right|^{2} d x+\frac{\lambda}{\mu} \int_{A_{n}}\left(f\left(x, u_{n}\right) u_{n}-\mu F\left(x, u_{n}\right)\right) d x \\
\geq & \kappa\left(V^{-}-M\right) r^{2} \operatorname{meas}\left(B_{i}\right)-\frac{2 \lambda}{\mu}(\mu+1) S_{q}\left\|h_{0}\right\|_{L^{q(x) /(q(x)-1)}\left(\mathbb{R}^{N}\right)}\left\|u_{n}\right\|_{W} \\
& -2 \frac{\lambda}{\mu}(\mu+1) \sum_{i=1}^{m}\left\|h_{i}\right\|_{L^{q(x) /\left(q(x)-\gamma_{i}(x)\right)}\left(\mathbb{R}^{N}\right)} \mathcal{C}(r)\left(\left\|u_{n}\right\|_{W}^{2 /\left(\left(q / \gamma_{i}\right)^{+}\right)}+\left\|u_{n}\right\|_{W}^{2 /\left(\left(q / \gamma_{i}\right)^{-}\right)}\right)
\end{aligned}
$$

for $i=1,2$. Combining (4.2) and (4.5), we have

$$
\begin{aligned}
& C\left(1+\left\|u_{n}\right\|_{W}\right) \\
\geq & \frac{1}{n_{0}}\left(\frac{1}{2}-\frac{1}{\mu}\right)\left\|u_{n}\right\|_{W}^{2}+\kappa\left(V^{-}-M\right) r^{2} \operatorname{meas}\left(B_{i}\right) \\
& -\frac{2 \lambda}{\mu}(\mu+1) S_{q}\left\|h_{0}\right\|_{L^{q(x) /(q(x)-1)\left(\mathbb{R}^{N}\right)}}\left\|u_{n}\right\|_{W} \\
& -2 \frac{\lambda}{\mu}(\mu+1) \sum_{i=1}^{m}\left\|h_{i}\right\|_{L^{q(x) /\left(q(x)-\gamma_{i}(x)\right)}\left(\mathbb{R}^{N}\right)} \mathcal{C}(r)\left(\left\|u_{n}\right\|_{W}^{2 /\left(\left(q / \gamma_{i}\right)^{+}\right)}+\left\|u_{n}\right\|_{W}^{2 /\left(\left(q / \gamma_{i}\right)^{-}\right)}\right)
\end{aligned}
$$

for $i=1$ or $i=2$. By hypothesis $\left(q / \gamma_{i}\right)^{+} \geq\left(q / \gamma_{i}\right)^{-}>1$, from 4.6$)$, we conclude that the sequence $\left\{u_{n}\right\}$ is bounded in $W$. The remaining proofs are the same in Lemma 3.4, we 
only need to check

$$
\int_{\mathbb{R}^{N}}\left|\left(f\left(x, u_{n}\right)-f(x, u)\right)\left(u_{n}-u\right)\right| d x \rightarrow 0 \quad \text { as } n \rightarrow \infty .
$$

Indeed, we have

$$
\begin{aligned}
& \int_{\mathbb{R}^{N}}\left|f\left(x, u_{n}\right) \|\left(u_{n}-u\right)\right| d x \\
\leq & 2\left\|h_{0}\right\|_{L^{q(x) /(q(x)-1)\left(\mathbb{R}^{N}\right)}}\left\|u_{n}-u\right\|_{L^{q(x)}\left(\mathbb{R}^{N}\right)} \\
& +3 \sum_{i=1}^{m}\left\|h_{i}\right\|_{L^{q(x) /\left(q(x)-\gamma_{i}(x)\right)}\left(\mathbb{R}^{N}\right)} \max \left\{\left(\max \left\{\left\|u_{n}\right\|_{L^{q(x)}\left(\mathbb{R}^{N}\right)}^{q^{+}},\left\|u_{n}\right\|_{L^{q(x)}\left(\mathbb{R}^{N}\right)}^{q^{-}}\right\}\right)^{1 /\left(q / \gamma_{i}\right)^{+}},\right. \\
& \left.\left(\max \left\{\left\|u_{n}\right\|_{L^{q(x)}\left(\mathbb{R}^{N}\right)}^{q^{+}},\left\|u_{n}\right\|_{L^{q(x)}\left(\mathbb{R}^{N}\right)}^{q^{-}}\right\}\right)^{1 /\left(q / \gamma_{i}\right)^{-}}\right\}\left\|u_{n}-u\right\|_{L^{q(x)}\left(\mathbb{R}^{N}\right)} \\
+ & g_{m+1}\left\|u_{n}\right\|_{L^{2}\left(\mathbb{R}^{N}\right)}\left\|u_{n}-u\right\|_{L^{2}\left(\mathbb{R}^{N}\right)} \\
+ & \max \left\{\left(\max \left\{\left\|u_{n}\right\|_{L^{q(x)}\left(\mathbb{R}^{N}\right)}^{q^{+}},\left\|u_{n}\right\|_{L^{q(x)}\left(\mathbb{R}^{N}\right)}^{q^{-}}\right\}\right)^{1 /(q /(q-1))^{+}},\right. \\
& \left.\left(\max \left\{\left\|u_{n}\right\|_{L^{q(x)}\left(\mathbb{R}^{N}\right)}^{q^{+}},\left\|u_{n}\right\|_{L^{q(x)}\left(\mathbb{R}^{N}\right)}^{q^{-}}\right\}\right)^{1 /(q /(q-1))^{-}}\right\}\left\|u_{n}-u\right\|_{L^{q(x)}\left(\mathbb{R}^{N}\right)}
\end{aligned}
$$

and

$$
\begin{aligned}
& \int_{\mathbb{R}^{N}}\left|f(x, u) \|\left(u_{n}-u\right)\right| d x \\
\leq & 2\left\|h_{0}\right\|_{L^{q(x) /(q(x)-1)\left(\mathbb{R}^{N}\right)}}\left\|u_{n}-u\right\|_{L^{q(x)}\left(\mathbb{R}^{N}\right)} \\
+ & 3 \sum_{i=1}^{m}\left\|h_{i}\right\|_{L^{q(x) /\left(q(x)-\gamma_{i}(x)\right)}\left(\mathbb{R}^{N}\right)} \max \left\{\left(\max \left\{\|u\|_{L^{q(x)}\left(\mathbb{R}^{N}\right)}^{q^{+}},\|u\|_{L^{q(x)}\left(\mathbb{R}^{N}\right)}^{q^{-}}\right\}\right)^{1 /\left(q / \gamma_{i}\right)^{+}},\right. \\
& \left.\left(\max \left\{\|u\|_{L^{q(x)}\left(\mathbb{R}^{N}\right)}^{q^{+}},\|u\|_{L^{q(x)}\left(\mathbb{R}^{N}\right)}^{q^{-}}\right\}\right)^{1 /\left(q / \gamma_{i}\right)^{-}}\right\}\left\|u_{n}-u\right\|_{L^{q(x)}\left(\mathbb{R}^{N}\right)} \\
+ & g_{m+1}\|u\|_{L^{2}\left(\mathbb{R}^{N}\right)}\left\|u_{n}-u\right\|_{L^{2}\left(\mathbb{R}^{N}\right)} \\
+ & \max \left\{\left(\max \left\{\|u\|_{L^{q(x)}\left(\mathbb{R}^{N}\right)}^{q^{+}},\|u\|_{L^{q(x)}\left(\mathbb{R}^{N}\right)}^{q^{-}}\right\}\right)^{1 /(q /(q-1))^{+}},\right. \\
& \left.\left(\max \left\{\|u\|_{L^{q(x)}\left(\mathbb{R}^{N}\right)}^{q^{+}},\|u\|_{L^{q(x)}\left(\mathbb{R}^{N}\right)}^{q^{-}}\right\}\right)^{1 /(q /(q-1))^{-}}\right\}\left\|u_{n}-u\right\|_{L^{q(x)}\left(\mathbb{R}^{N}\right) .}
\end{aligned}
$$

Combining (4.8) and 4.9), the fact that $u_{n} \rightarrow u$ strongly in $L^{2}\left(\mathbb{R}^{N}\right)$ and $L^{q(x)}\left(\mathbb{R}^{N}\right)$, we deduce 4.7 .

Since $W$ is a Hilbert space, then we get the following result.

Corollary 4.2. There exists a basic orthonormal $\left\{e_{1}, e_{2}, \ldots, e_{n}, \ldots\right\}$ of $W$ such that

$$
W^{*}=\left\{e_{1}^{*}, e_{2}^{*}, \ldots, e_{n}^{*}, \ldots\right\} .
$$

Denote $X_{i}=\mathbb{R} e_{i}$, we have $W=\overline{\bigoplus_{i=1}^{\infty} X_{i}}$. 
Following the notation used by Bartsch [5, Theorem 2.5], in the sequel for any $k \in \mathbb{N}$, we put

$$
Y_{k}:=\operatorname{span}\left\{e_{1}, \ldots, e_{k}\right\} \quad \text { and } \quad Z_{k}:=\overline{\operatorname{span}\left\{e_{k}, e_{k+1}, \ldots\right\}} .
$$

Since $Y_{k}$ is finite-dimensional, all norms on $Y_{k}$ are equivalent. Therefore, there exist two positive constants $C_{k, q}$ and $\widetilde{C}_{k, q}$, depending on $k, q$, such that for any $u \in Y_{k}$

$$
C_{k, q}\|u\|_{W} \leq\|u\|_{L^{q}\left(\mathbb{R}^{N}\right)} \leq \widetilde{C}_{k, q}\|u\|_{W}
$$

for $q=2$ or any $q \in\left(2,2_{s}^{*}(x)\right), q \in C_{+}\left(\mathbb{R}^{N}\right)$ is a uniformly continuous function. The Fountain Theorem provides the existence of an unbounded sequence of critical value for a smooth functional, under suitable compactness condition and geometry assumption on it, which, in our framework, read as follows:

(i) $a_{k}:=\max \left\{I(u): u \in Y_{k},\|u\|_{W}=r_{k}\right\} \leq 0$,

(ii) $b_{k}:=\inf \left\{I(u): u \in Z_{k},\|u\|_{W}=\alpha_{k}\right\} \rightarrow \infty$ as $k \rightarrow \infty$.

Now, we prove Theorem 1.5. The idea consists in applying the Fountain Theorem. By Lemma 4.1, we see that $I$ satisfies the Palais-Smale condition and the condition $\left(f_{5}\right)$ implies $I(-u)=I(u)$ for any $u \in W$. Then, it remains to show the geometry condition for the function $I$. To this purpose, let us proceed by steps.

Step 1 . For any $k \in \mathbb{N}$, there exists $r_{k}>0$ large enough such that

$$
a_{k}:=\max \left\{I(u): u \in Y_{k},\|u\|_{W}=r_{k}\right\} \leq 0 .
$$

By arguments as in Lemma 3.3, we have $I(u) \rightarrow-\infty$ as $r_{k} \rightarrow+\infty$. We complete Step 1.

Step 2. For any $k \in \mathbb{N}$, denote

$$
\beta_{k}:=\sup \left\{\|u\|_{L^{q(x)}\left(\mathbb{R}^{N}\right)}: u \in Z_{k},\|u\|_{W}=1\right\}
$$

then $\beta_{k} \rightarrow 0$ as $k \rightarrow \infty$. Indeed, suppose that $\beta_{k} \not \rightarrow 0$ as $k \rightarrow \infty$, then there exist $\varepsilon_{0}>0$ and $\left\{u_{i}\right\} \subset W$ with $u_{i}$ being orthogonal with $Y_{k_{i}-1}$ such that $\left\|u_{i}\right\|=1,\left\|u_{i}\right\|_{L^{q(x)}\left(\mathbb{R}^{N}\right)} \geq$ $\varepsilon_{0}$, where $k_{i} \rightarrow \infty$ as $i \rightarrow \infty$. For any $v \in W$, we can write $v=\sum_{i=1}^{\infty}\left\langle v, e_{i}\right\rangle e_{i}$ and $\lim _{n \rightarrow \infty} \sum_{i=1}^{n}\left\langle v, e_{i}\right\rangle e_{i}=v$ strongly in $W$. Therefore, we can find $w_{i} \in Y_{k_{i}-1}$ such that $w_{i} \rightarrow v$ as $i \rightarrow \infty$. Hence,

$$
\left|\left\langle u_{i}, v\right\rangle\right|=\left|\left\langle u_{i}, w_{i}-v\right\rangle\right| \leq\left\|u_{i}\right\|_{W}\left\|w_{i}-v\right\|_{W} \rightarrow 0
$$

as $i \rightarrow \infty$. Then $u_{i} \rightarrow 0$ weakly in $W$. By Lemmas 2.6 and 2.9, we get $u_{i} \rightarrow 0$ in $L^{q(x)}\left(\mathbb{R}^{N}\right)$, which contradicts with $\left\|u_{i}\right\|_{L^{q(x)}\left(\mathbb{R}^{N}\right)} \geq \varepsilon_{0}>0$ for all $i$. Thus, we must have $\beta_{k} \rightarrow 0$ as $k \rightarrow \infty$. Similarly, we also have

$$
\beta_{k}^{*}:=\sup \left\{\|u\|_{L^{2}\left(\mathbb{R}^{N}\right)}: u \in Z_{k},\|u\|_{W}=1\right\} \rightarrow 0
$$


as $k \rightarrow \infty$.

Step 3. There exists $\alpha_{k}>0$ such that

$$
b_{k}:=\inf \left\{I(u): u \in Z_{k},\|u\|_{W}=\alpha_{k}\right\} \rightarrow+\infty
$$

as $k \rightarrow+\infty$. From 2.13 , we have

$$
\begin{aligned}
I(u) \geq & \frac{1}{2}\|u\|_{W}^{2}-2 \lambda\left\|h_{0}\right\|_{L^{q(x) /(q(x)-1)}}\|u\|_{L^{q(x)}\left(\mathbb{R}^{N}\right)}-\lambda g_{m+1}\|u\|_{L^{2}\left(\mathbb{R}^{N}\right)}^{2} \\
& -2 \lambda \sum_{i=1}^{m}\left\|h_{i}\right\|_{L^{q(x) /\left(q(x)-\gamma_{i}(x)\right)}\left(\mathbb{R}^{N}\right)} \max \left\{\left(\max \left\{\|u\|_{L^{q(x)}\left(\mathbb{R}^{N}\right)}^{q^{+}},\|u\|_{L^{q(x)}\left(\mathbb{R}^{N}\right)}^{q^{-}}\right\}\right)^{1 /\left(q / \gamma_{i}\right)^{+}},\right. \\
& \left.\quad\left(\max \left\{\|u\|_{L^{q(x)}\left(\mathbb{R}^{N}\right)}^{q^{+}},\|u\|_{L^{q(x)}\left(\mathbb{R}^{N}\right)}^{q^{-}}\right\}\right)^{1 /\left(q / \gamma_{i}\right)^{-}}\right\} \\
& -h_{m+1} \lambda \max \left\{\|u\|_{L^{q(x)}\left(\mathbb{R}^{N}\right)}^{q^{+}},\|u\|_{L^{q(x)}\left(\mathbb{R}^{N}\right)}^{q^{-}}\right\} .
\end{aligned}
$$

Then we get

$$
\begin{aligned}
I(u) \geq & \frac{1}{2}\|u\|_{W}^{2}-2 \lambda\left\|h_{0}\right\|_{L^{q(x) /(q(x)-1)}\left(\mathbb{R}^{N}\right)}\|u\|_{L^{q(x)}\left(\mathbb{R}^{N}\right)}-\lambda g_{m+1}\|u\|_{L^{2}\left(\mathbb{R}^{N}\right)}^{2} \\
& -2 \lambda \sum_{i=1}^{m}\left\|h_{i}\right\|_{L^{q(x) /\left(q(x)-\gamma_{i}(x)\right)}\left(\mathbb{R}^{N}\right)}\left\{\left(\|u\|_{L^{q(x)}\left(\mathbb{R}^{N}\right)}^{q^{+}}+\|u\|_{L^{q(x)}\left(\mathbb{R}^{N}\right)}^{q^{-}}\right)^{1 /\left(q / \gamma_{i}\right)^{+}}\right. \\
& \left.+\left(\|u\|_{L^{q(x)}\left(\mathbb{R}^{N}\right)}^{q^{+}}+\|u\|_{L^{q(x)}\left(\mathbb{R}^{N}\right)}^{q^{-}}\right)^{1 /\left(q / \gamma_{i}\right)^{-}}\right\} \\
& -h_{m+1} \lambda\left(\|u\|_{L^{q(x)}\left(\mathbb{R}^{N}\right)}^{q^{+}}+\|u\|_{L^{q(x)}\left(\mathbb{R}^{N}\right)}^{q^{-}}\right) .
\end{aligned}
$$

Now, we show that there exist uniqueness $t_{1}\left(\beta_{k}\right)>0$ such that

$$
h\left(t_{1}\left(\beta_{k}\right)\right)=\frac{1}{A \lambda h_{m+1}},
$$

where $A>2$ is a constant and

$$
h(t)=h_{1}(t)-\frac{1}{A \lambda h_{m+1}}, \quad t \geq 0,
$$

where $h_{1}(t)=\beta_{k}^{q^{+}} t^{q^{+}-2}+\beta_{k}^{q^{-}} t^{q^{-}-2}$. Indeed, we have $h(0)=-1 /\left(A \lambda h_{m+1}\right)<0$ and $\lim _{t \rightarrow+\infty} h(t)=+\infty$ for each $k$. Then there there exists $t_{0}(k) \gg 0$ such that $h\left(t_{0}(k)\right)>$ 0 . By the mean-value theorem for continuous function, there exists $t_{1}\left(\beta_{k}\right)$ such that $h\left(t_{1}\left(\beta_{k}\right)\right)=0$ which proves 4.11). Since $h^{\prime}(t)=\beta_{k}^{q^{+}}\left(q^{+}-2\right) t^{q^{+}-3}+\beta_{k}^{q^{-}}\left(q^{-}-2\right) t^{q^{-}-3}>0$ for all $t \in(0,+\infty)$, then $h(t)$ is a increasing function in $[0,+\infty)$. This means that $t_{1}\left(\beta_{k}\right)$ is uniqueness. We see that $\lim _{k \rightarrow+\infty} t_{1}\left(\beta_{k}\right)=+\infty$. Conversely, if there exists $B>0$ such that $t_{1}\left(\beta_{k}\right) \leq B$ for all $k \geq 1$, then $\lim _{k \rightarrow+\infty}\left(\beta_{k}^{q^{+}}\left(t_{1}\left(\beta_{k}\right)\right)^{q^{+}-2}+\beta_{k}^{q^{-}}\left(t_{1}\left(\beta_{k}\right)\right)^{q^{-}-2}\right)=0$. It is a contradiction since $\lim _{k \rightarrow+\infty} h_{1}\left(t_{1}\left(\beta_{k}\right)\right)=\frac{1}{A \lambda h_{m+1}}>0$. Then for $u \in W$ such that 
$\|u\|_{W}=\alpha_{k}=t_{1}\left(\beta_{k}\right) \rightarrow+\infty$ as $k \rightarrow+\infty$, and we have

$$
\begin{aligned}
I(u) \geq & \frac{1}{2}\|u\|_{W}^{2}-2 \lambda \beta_{k}\left\|h_{0}\right\|_{L^{q(x) /(q(x)-1)}}\|u\|_{W}-\lambda g_{m+1}\left(\beta_{k}^{*}\right)^{2}\|u\|_{W}^{2} \\
& -2 \lambda \sum_{i=1}^{m}\left\|h_{i}\right\|_{L^{q(x) /\left(q(x)-\gamma_{i}(x)\right)}\left(\mathbb{R}^{N}\right)} \\
& \quad \times\left\{\left(\beta_{k}^{q^{+}}\|u\|_{W}^{q^{+}}+\beta_{k}^{q^{-}}\|u\|_{W}^{q^{-}}\right)^{1 /\left(q / \gamma_{i}\right)^{+}}+\left(\beta_{k}^{q^{+}}\|u\|_{W}^{q^{+}}+\beta_{k}^{q^{-}}\|u\|_{W}^{q^{-}}\right)^{1 /\left(q / \gamma_{i}\right)^{-}}\right\} \\
& -h_{m+1} \lambda\left(\beta_{k}^{q^{+}}\|u\|_{W}^{q^{+}}+\beta_{k}^{q^{-}}\|u\|_{W}^{q^{-}}\right) .
\end{aligned}
$$

Therefore, we obtain

$$
\begin{aligned}
& I(u) \geq\|u\|_{W}^{2}\left(\frac{1}{2}-g_{m+1} \lambda \beta_{k}^{* 2}-h_{m+1} \lambda\left(\beta_{k}^{q^{+}}\|u\|_{W}^{q^{+}-2}+\beta_{k}^{q^{-}}\|u\|_{W}^{q^{-}-2}\right)\right) \\
& -2 \lambda \sum_{i=1}^{m}\left\|h_{i}\right\|_{L^{q(x) /\left(q(x)-\gamma_{i}(x)\right)}\left(\mathbb{R}^{N}\right)} \\
& \times\left\{\left(\beta_{k}^{q^{+}}\|u\|_{W}^{q^{+}}+\beta_{k}^{q^{-}}\|u\|_{W}^{q^{-}}\right)^{1 /\left(q / \gamma_{i}\right)^{+}}+\left(\beta_{k}^{q^{+}}\|u\|_{W}^{q^{+}}+\beta_{k}^{q^{-}}\|u\|_{W}^{q^{-}}\right)^{1 /\left(q / \gamma_{i}\right)^{-}}\right\} \\
& =\left(\frac{1}{2}-\frac{1}{A}-g_{m+1} \lambda \beta_{k}^{* 2}\right) \alpha_{k}^{2} \\
& -2 \lambda \sum_{i=1}^{m}\left\|h_{i}\right\|_{L^{q(x) /\left(q(x)-\gamma_{i}(x)\right)}\left(\mathbb{R}^{N}\right)}\left(\left(\frac{\alpha_{k}^{2}}{A \lambda h_{m+1}}\right)^{1 /\left(q / \gamma_{i}\right)^{+}}+\left(\frac{\alpha_{k}^{2}}{A \lambda h_{m+1}}\right)^{1 /\left(q / \gamma_{i}\right)^{-}}\right),
\end{aligned}
$$

where $\beta_{k}$ and $\beta_{k}^{*}$ are defined in Step 2. By the assumption $\inf _{x \in \mathbb{R}^{N}}\left(q(x)-\gamma_{i}(x)\right)>0$ for all $i=1, \ldots, m$, then $\left(q / \gamma_{i}\right)^{+} \geq\left(q / \gamma_{i}\right)^{-}>1$ and above inequality implies $\lim _{k \rightarrow+\infty} I(u)=$ $+\infty$. Hence, all the geometric features of the Fountain Theorem are satisfied, then $I$ has an unbounded sequence of critical values which are week solutions of problem (1.1).

\section{Proof of Theorem 1.6}

Let $X$ be a real Banach space. Set

$$
\mathcal{R}=\{E \in X \backslash\{0\}: E \text { is compact and } E=-E\}
$$

Definition 5.1. Let $E \in \mathcal{R}$ and $X=\mathbb{R}^{k}$. The genus $\gamma(E)$ of $E$ is defined by

$$
\gamma(E)=\min \left\{k \geq 1: \text { there exists an odd continuous mapping } \phi: E \rightarrow \mathbb{R}^{k} \backslash\{0\}\right\} \text {. }
$$

If such a mapping does not exist for any $k>0$, we set $\gamma(E)=\infty$. Note that if $E$ is a subset which consists of finitely many pairs of points, then $\gamma(E)=1$. For the sake of convenience, $\gamma(\emptyset)=0$. A typical example of a set of genus $k$ is a set, which is homeomorphic to a $(k-1)$-dimensional sphere via an odd map. Now, we give some results of Krasnoselskii's genus 19, which are necessary throughout this section. 
Lemma 5.2. Let $X=\mathbb{R}^{N}$ and $\partial \Omega$ be the boundary of an open, symmetric and bounded subset $\Omega \subset \mathbb{R}^{N}$ with $0 \in \Omega$. Then $\gamma(\partial \Omega)=N$.

It follows from Lemma 5.2 that $\gamma\left(S^{N-1}\right)=N$. Now we present the results due to Clarke 7].

Theorem 5.3. Let $G \in C^{1}(X, \mathbb{R})$ be functional satisfying the (PS) condition. Furthermore, let us suppose that

(i) $G$ is bounded from below and even;

(ii) there is a compact set $K \subset \mathcal{R}$ such that $\gamma(K)=k$ and $\sup _{x \in K} G(x)<G(0)$.

Then $G$ possesses at least $k$ pairs of distinct critical points, and their corresponding critical values are less than $G(0)$.

Now, we prove Theorem 1.6. By the condition $\left(f_{5}\right)$, we see that $I$ is an even function on $W$. We have

$$
\begin{aligned}
I(u) \geq & \frac{1}{2}\|u\|_{W}^{2}-2 \lambda\left\|h_{0}\right\|_{L^{1}\left(\mathbb{R}^{N}\right)}\|u\|_{L^{2}\left(\mathbb{R}^{N}\right)} \\
& -2 \lambda \sum_{i=1}^{m}\left\|h_{i}\right\|_{L^{2 /\left(2-\gamma_{i}(x)\right)}\left(\mathbb{R}^{N}\right)}\left\{\|u\|_{L^{2}\left(\mathbb{R}^{N}\right)}^{2 /\left(2 / \gamma_{i}\right)^{+}}+\|u\|_{L^{2}\left(\mathbb{R}^{N}\right)}^{2 /\left(2 / \gamma_{i}\right)^{-}}\right\} \\
\geq & \frac{1}{2}\|u\|_{W}^{2}-2 \lambda S_{2}\left\|h_{0}\right\|_{L^{1}\left(\mathbb{R}^{N}\right)}\|u\|_{W} \\
& -2 \lambda \kappa_{*} \sum_{i=1}^{m}\left\|h_{i}\right\|_{L^{2 /\left(2-\gamma_{i}(x)\right)\left(\mathbb{R}^{N}\right)}}\left\{\|u\|_{W}^{2 /\left(2 / \gamma_{i}\right)^{+}}+\|u\|_{W}^{2 /\left(2 / \gamma_{i}\right)^{-}}\right\},
\end{aligned}
$$

where $\kappa_{*}>0$ is a suitable constant. From the assumptions $\left(2 / \gamma_{i}\right)^{+} \geq\left(2 / \gamma_{i}\right)^{-}>1$, then (5.1) implies that $\lim _{\|u\|_{W} \rightarrow+\infty} I(u)=+\infty$. This means that $I$ is bounded from below. Hence, any (PS) sequence of $I$ is bounded. By arguments as in Lemma 4.1, we have that $I$ satisfies the (PS) condition. By Theorem 5.3, we need check the condition (ii) for $I$. We consider the subspace $Y_{k}$ of $W$ generated by the $k$ vectors $\left\{e_{1}, e_{2}, \ldots, e_{k}\right\}$. For $\rho>0$, consider

$$
K_{k}(\rho)=\left\{u \in Y_{k}:\|u\|_{W}^{2}=\sum_{i=1}^{k} \xi_{i}^{2}=\rho^{2}\right\},
$$

where $u=\sum_{i=1}^{k} \xi_{i} e_{i}$. Then $K_{k}(\rho)$ is a compact subset of $W$. Since $Y_{k}$ is finite dimensional, then all norms are equivalent, we can choose $\rho$ small enough such that $\|u\|_{L^{\infty}\left(\mathbb{R}^{N}\right)} \leq$ $\mathcal{C}\|u\|_{W} \leq \mathcal{C} \rho \leq \eta$, where $\mathcal{C}>0$ is a suitable constant. We have

$$
I(u)=\frac{1}{2}\|u\|_{W}^{2}-\lambda \int_{\mathbb{R}^{N}} F(x, u) d x \leq \frac{1}{2}\|u\|_{W}^{2}-\lambda \delta \int_{\mathbb{R}^{N}}|u|^{2} d x
$$


for $u \in Y_{k}$. From (4.10, we have

$$
\int_{\mathbb{R}^{N}}|u|^{2} d x \geq C_{k, 2}^{2}\|u\|_{W}^{2}
$$

Combining 5.2 and 5.3 , there exists $\lambda_{0}=\frac{1}{2 \delta C_{k, 2}^{2}}$ such that

$$
I(u) \leq\left(\frac{1}{2}-\lambda \delta C_{k, 2}^{2}\right)\|u\|_{W}^{2}<0
$$

for all $u \in Y_{k},\|u\|_{W}=\rho$ and $\lambda \in\left(\lambda_{0},+\infty\right)$. Since $I$ is continuous function on $W$ and $K_{k}(\rho)$ is a compact subset of $W$, then we have

$$
\sup _{u \in K_{k}(\rho)} I(u)<0=I(0)
$$

On the other hand, we consider the following odd homeomorphism $\chi: K_{k}(\rho) \rightarrow S^{k-1}$ defined as $\chi(u)=\left(\xi_{1}, \ldots, \xi_{k}\right)$, where $S^{k-1}$ is the sphere in $\mathbb{R}^{k}$. It follows from Lemma 5.2 that $\gamma\left(S^{k-1}\right)=k$. Thanks to Theorem 5.3, $I$ possesses at least $k$ pairs of distinct critical points, and their corresponding critical values are less than $I(0)=0$ which are weak solutions of problem (1.1).

\section{Acknowledgments}

The author wishes to thank the referee for a very careful reading of the manuscript, and for pointing out misprints that led to the improvement of the original manuscript.

\section{References}

[1] C. O. Alves and S. Liu, On superlinear $p(x)$-Laplacian equations in $\mathbb{R}^{N}$, Nonlinear Anal. 73 (2010), no. 8, 2566-2579.

[2] E. Azroul, A. Benkirane and M. Shimi, Eigenvalue problems involving the fractional $p(x)$-Laplacian operator, Adv. Oper. Theory. 4 (2019), no. 2, 539-555.

[3] A. Bahrouni, Comparison and sub-supersolution principles for the fractional $p(x)$ Laplacian, J. Math. Anal. Appl. 458 (2018), no. 2, 1363-1372.

[4] A. Bahrouni and V. D. Rădulescu, On a new fractional Sobolev space and applications to nonlocal variational problems with variable exponent, Discrete Contin. Dyn. Syst. Ser. S 11 (2018), no. 3, 379-389.

[5] T. Bartsch, Infinitely many solutions of a symmetric Dirichlet problem, Nonlinear Anal. 20 (1993), no. 10, 1205-1216. 
[6] N. T. Chung, Eigenvalue problems for fractional $p(x, y)$-Laplacian equations with indefinite weight, Taiwanese J. Math. 23 (2019), no. 5, 1153-1173.

[7] D. C. Clark, A variant of the Lusternik-Schnirelman theory, Indiana Univ. Math. J. 22 (1972), 65-74.

[8] L. Diening, P. Harjulehto, P. Hästö and M. Růžička, Lebesgue and Sobolev Spaces with Variable Exponents, Lecture Notes in Mathematics 2017, Springer, Heidelberg, 2011.

[9] X. Fan and D. Zhao, On the spaces $L^{p(x)}(\Omega)$ and $W^{m, p(x)}(\Omega)$, J. Math. Anal. Appl. 263 (2001), no. 2, 424-446.

[10] K. Ho and Y. H. Kim, A-priori bounds and multiplicity of solutions for nonlinear elliptic problems involving the fractional $p(\cdot)$-Laplacian, Nonlinear Anal. 188 (2019), 179-201.

[11] U. Kaufmann, J. D. Rossi and R. Vidal, Fractional Sobolev spaces with variable exponents and fractional $p(x)$-Laplacians, Electron. J. Qual. Theory Differ. Equ. 2017 (2017), no. 76, 10 pp.

[12] K. Kikuchi and A. Negoro, On Markov process generated by pseudodifferentail operator of variable order, Osaka J. Math. 34 (1997), no. 2, 319-335.

[13] O. Kováčik and J. Rákosník, On spaces $L^{p(x)}$ and $W^{k, p(x)}$, Czechoslovak Math. J. 41 (1991), no. 4, 592-618.

[14] N. Laskin, Fractional quantum mechanics and Lévy path integrals, Phys. Lett. A 268 (2000), no. 4-6, 298-305.

[15] H.-G. Leopold, Embedding of function spaces of variable order of differentiation in function spaces of variable order of integration, Czechoslovak Math. J. 49 (1999), no. 3, 633-644.

[16] C. F. Lorenzo and T. T. Hartley, Variable order and distributed order fractional operators, Nonlinear Dynam. 29 (2002), no. 1-4, 57-98.

[17] G. Molica Bisci, V. D. Radulescu and R. Servadei, Variational Methods for Nonlocal Fractional Problems, Encyclopedia of Mathematics and its Applications 162, Cambridge University Press, Cambridge, 2016.

[18] P. Pucci, M. Xiang and B. Zhang, Multiple solutions for nonhomogeneous Schrödinger-Kirchhoff type equations involving the fractional p-Laplacian in $\mathbb{R}^{N}$, Calc. Var. Partial Differential Equations 54 (2015), no. 3, 2785-2806. 
[19] P. H. Rabinowitz, Minimax Methods in Critical Point Theory with Applications to Differential Equations, CBMS Regional Conference Series in Mathematics 65, American Mathematical Society, Providence, RI, 1986.

[20] M. D. Ruiz-Medina, V. V. Anh and J. M. Angulo, Fractional generalized random fields of variable order, Stochastic Anal. Appl. 22 (2004), no. 3, 775-799.

[21] M. Struwe, Variational Methods: Applications to nonlinear partial differential equations and Hamiltonian systems, Springer-Verlag, Berlin, 1990.

[22] M. Willem, Minimax Theorems, Progress in Nonlinear Differential Equations and their Applications 24, Birkhäuser Boston, Boston, MA, 1996.

[23] M. Xiang, B. Zhang and D. Yang, Multiplicity results for variable-order fractional Laplacian equations with variable growth, Nonlinear Anal. 178 (2019), 190-204.

Nguyen Van Thin

Department of Mathematics, Thai Nguyen University of Education, Luong Ngoc Quyen street, Thai Nguyen city, Thai Nguyen, Vietnam

and

Thang Long Institute of Mathematics and Applied Sciences, Thang Long University, Nghiem Xuan Yem, Hoang Mai, Hanoi, Vietnam

E-mail addresses: thinmath@gmail.com, thinnv@tnue.edu.vn 